\title{
Calabi-Yau Manifolds, Hermitian Yang-Mills Instantons, and Mirror Symmetry
}

\author{
Hyun Seok Yang ${ }^{1}$ and Sangheon Yun ${ }^{2}$ \\ ${ }^{1}$ Center for Quantum Spacetime, Sogang University, Seoul 121-741, Republic of Korea \\ ${ }^{2}$ Institute for the Early Universe, Ewha Womans University, Seoul 120-750, Republic of Korea \\ Correspondence should be addressed to Hyun Seok Yang; ya19683@gmail.com
}

Received 24 October 2016; Revised 9 April 2017; Accepted 30 July 2017; Published 9 November 2017

Academic Editor: Anna Cimmino

Copyright (C) 2017 Hyun Seok Yang and Sangheon Yun. This is an open access article distributed under the Creative Commons Attribution License, which permits unrestricted use, distribution, and reproduction in any medium, provided the original work is properly cited. The publication of this article was funded by SCOAP ${ }^{3}$.

\begin{abstract}
We address the issue of why Calabi-Yau manifolds exist with a mirror pair. We observe that the irreducible spinor representation of the Lorentz group Spin(6) requires us to consider the vector spaces of two forms and four forms on an equal footing. The doubling of the two-form vector space due to the Hodge duality doubles the variety of six-dimensional spin manifolds. We explore how the doubling is related to the mirror symmetry of Calabi-Yau manifolds. Via the gauge theory formulation of six-dimensional Riemannian manifolds, we show that the curvature tensor of a Calabi-Yau manifold satisfies the Hermitian Yang-Mills equations on the Calabi-Yau manifold. Therefore, the mirror symmetry of Calabi-Yau manifolds can be recast as the mirror pair of Hermitian Yang-Mills instantons. We discuss the mirror symmetry from the gauge theory perspective.
\end{abstract}

\section{Introduction}

String theory predicts [1] that six-dimensional Riemannian manifolds have to play an important role in explaining our four-dimensional world. They serve as an internal geometry of string theory with six extra dimensions and their shapes and topology determine a detailed structure of the multiplets for elementary particles and gauge fields through the Kaluza-Klein compactification. This program, initiated by a classic paper [2], tries to make contact with a lowenergy phenomenology in our four-dimensional world. In particular, a Calabi-Yau (CY) manifold is a (compact) Kähler manifold with vanishing Ricci curvature and so a vacuum solution of the Einstein equations. They have a prominent role in superstring theory and have been a central focus in both contemporary mathematics and mathematical physics. As the holonomy group of CY manifolds is $S U(3)$, the compactification onto a CY manifold in heterotic superstring theory preserves $\mathcal{N}=1$ supersymmetry in four dimensions. One of the most interesting features in the CY compactification is that type II superstring theories compactified on two distinct CY manifolds lead to an identical effective field theory in four dimensions $[1,3]$. This suggests that CY manifolds exist with a mirror pair $(M, \widetilde{M})$ where the number of vector multiplets $h^{1,1}(M)$ on $M$ is the same as the number of hypermultiplets $h^{2,1}(\widetilde{M})$ on $\widetilde{M}$ and vice versa. Here $h^{p, q}(M)=\operatorname{dim} H^{p, q}(M)$ is the Hodge number of a CY manifold $M$. This duality between two CY manifolds is known as the mirror symmetry [3]. While many beautiful properties of the mirror symmetry have been discovered and it has been even proven for some cases, it is fair to say that we are still far away from a deep understanding for the origin of mirror symmetry.

Mirror symmetry is a correspondence between two topologically distinct CY manifolds that give rise to exactly the same physical theory. To recapitulate the mirror symmetry, let $M$ be a compact $C Y$ manifold. The only nontrivial cohomology of the CY manifold is contained in $H^{1,1}(M)$ and $H^{2,1}(M)$ besides the one-dimensional cohomologies $h^{0,0}(M)=h^{3,3}(M)=h^{3,0}(M)=h^{0,3}(M)=1$. These cohomology classes parameterize CY moduli. It is known [3] that every $H^{1,1}(M)$, on one hand, is represented by a real closed $(1,1)$-form which forms a Kähler class represented by the Kähler form of a CY manifold $M$. The elements in 
$H^{1,1}(M)$ infinitesimally change the Kähler structure of the CY manifold and are therefore called Kähler moduli. (In string theory, these moduli are usually complexified by including $B$-field.) On the other hand, $H^{2,1}(M)$ parameterizes the complex structure moduli of a CY manifold $M$. It is thanks to the fact that the cohomology class of $(2,1)$-forms is isomorphic to the cohomology class $H_{\bar{\partial}}(T M)$, the first Dolbeault cohomology group of $M$ with values in a holomorphic tangent bundle TM that characterizes infinitesimal complex structure deformations. Hence the mirror symmetry of CY manifolds is the duality between two different CY 3-fold $M$ and $\widetilde{M}$ such that the Hodge numbers of $M$ and $\widetilde{M}$ satisfy the relations [3]

$$
\begin{aligned}
& h^{1,1}(M)=h^{2,1}(\widetilde{M}), \\
& h^{2,1}(M)=h^{1,1}(\widetilde{M}),
\end{aligned}
$$

or in a more general form

$$
h^{p, q}(M)=h^{3-p, q}(\widetilde{M})
$$

where the Hodge number $h^{p, q}$ of a CY manifold satisfies the relations $h^{p, q}=h^{q, p}$ and $h^{p, q}=h^{3-p, 3-q}$. As we have mentioned above, the only nontrivial deformations of a $\mathrm{CY}$ manifold are generated by the cohomology classes in $H^{1,1}(M)$ and $H^{2,1}(M)$ where $h^{1,1}(M)$ is the number of possible (in general, complexified) Kähler forms and $h^{2,1}(M)$ is the dimension of the complex structure moduli space of $M$. Mirror symmetry suggests that for each CY 3-fold $M$ there exists another CY 3-fold $\widetilde{M}$ whose Hodge numbers obey the relation (1).

From a physical point of view, two CY manifolds are related by mirror symmetry if the corresponding $\mathcal{N}=2$ superconformal field theories are mirror [4]. Two $\mathcal{N}=2$ superconformal field theories are said to be mirror if they are equivalent as quantum field theories. The mirror symmetry was interpreted mathematically by Kontsevich in his 1994 ICM talk as an equivalence of derived categories, dubbed the homological mirror symmetry [5]. The homological mirror symmetry states that the derived category of coherent sheaves on a Kähler manifold should be isomorphic to the Fukaya category of a mirror symplectic manifold [6]. The Fukaya category is described by the Lagrangian submanifold of a given symplectic manifold as its objects and the Floer homology groups as their morphisms. Hence the homological mirror symmetry formulates the mirror symmetry as an equivalence between certain aspects of complex geometry of a CY manifold and certain aspects of symplectic geometry of a mirror CY manifold in all dimensions. The geometric approach to mirror symmetry was also unveiled in [7]; that mirror symmetry is a geometric version of the Fourier-Mukai transformation along a dual special Lagrangian tori fibration on a mirror CY manifold which interchanges the symplectic geometry and the complex geometry of a mirror pair.

In this paper we will explore the gauge theory formulation of six-dimensional Riemannian manifolds to address the issue why CY manifolds exist with a mirror pair. In order to simplify an underlying argumentation, we will focus on orientable six-dimensional manifolds with spin structure. In general relativity, the Lorentz group appears as the structure group acting on orthonormal frames of the tangent bundle of a Riemannian manifold [8]. On the frame bundle, a Riemannian metric on spacetime manifold $M$ is replaced by a local orthonormal basis $E_{A}(A=1, \ldots, d)$ of the tangent bundle $T M$. Then Einstein gravity can be formulated as a gauge theory of Lorentz group where spin connections play a role of gauge fields and Riemann curvature tensors correspond to their field strengths. On a six-dimensional Riemannian manifold $M$, for example, local Lorentz transformations are orthogonal rotations in Spin(6), and spin connections $\omega_{A B}=$ $\omega_{M A B} d x^{M}$ are the spin(6)-valued gauge fields from the gauge theory point of view (we will use large letters to indicate a Lie group $G$ and small letters for its Lie algebra $\mathfrak{g}$ ). Then the Riemann curvature tensor $R_{A B}=d \omega_{A B}+\omega_{A C} \wedge \omega_{C B}$ precisely corresponds to the field strength of gauge fields $\omega_{A B}$ in Spin(6) gauge theory. Since the Lie group Spin(6) is isomorphic to $S U(4)$, the six-dimensional Euclidean gravity can be formulated as an $S U(4)$ Yang-Mills gauge theory. Via the gauge theory formulation of six-dimensional Riemannian manifolds, we want to identify gauge theory objects corresponding to CY manifolds and address their mirror symmetry from the perspective of Yang-Mills gauge theory. To understand why there exists a mirror pair of CY manifolds, in particular, we will employ the following well-known propositions for a $d$-dimensional Riemannian manifold $M$ :

(A) The Riemann curvature tensors $R_{A B}$ are $\operatorname{spin}(d)$ valued two forms in $\Omega^{2}(M)=\Lambda^{2} T^{*} M$.

(B) There exists a global isomorphism between $d$ dimensional Lorentz groups and classical Lie groups:

$$
\begin{aligned}
& \operatorname{Spin}(3) \cong S U(2), \\
& \operatorname{Spin}(4) \cong S U(2)_{L} \times S U(2)_{R}, \\
& \operatorname{Spin}(5) \cong S p(4), \\
& \operatorname{Spin}(6) \cong S U(4) .
\end{aligned}
$$

(C) There is an isomorphism between the Clifford algebra $\mathbb{C l}(d)$ in $d$-dimensions and the exterior algebra $\Lambda^{*} M$ of cotangent bundle $T^{*} M$ over $M[9,10]$ (the space of the Clifford algebra $\mathbb{C l}(d)$ is isomorphic, as a vector space, to the vector space of the exterior algebra $\Lambda^{*} M$. This is not, however, an isomorphism of associative algebras because the product in $\Lambda^{*} M$ is anticommutative while that in $\mathbb{C l}(d)$ is not due to the central term in the Dirac algebra (5)):

$$
\mathbb{C l}(d) \cong \Lambda^{*} M=\bigoplus_{k=0}^{d} \Omega^{k}(M),
$$

where $\Omega^{k}(M)=\Lambda^{k} T^{*} M$.

For the isomorphism (C) between the vector spaces, the "volume operator" $\Gamma_{d+1} \equiv \pm i^{d(d-1) / 2} \Gamma^{1} \cdots \Gamma^{d}$ in the Clifford 


\begin{tabular}{lcccccc}
\hline$M$ & $\operatorname{Spin}(6)$ & $S U(4)$ & & $\overline{\widetilde{M}}$ & $\operatorname{Spin}(6)$ & $S U(4)$ \\
\hline $\mathbb{A}: 3 \hookrightarrow 4$ & $\mathrm{CY} 3$ & $\mathrm{HYM}$ & $\Longleftrightarrow$ & $\mathbb{B}: \overline{3} \hookrightarrow \overline{4}$ & $\widetilde{\mathrm{CY} 3}$ & $\widetilde{\mathrm{HYM}}$ \\
$\mathbb{B}: \overline{3} \hookrightarrow \overline{4}$ & $\mathrm{CY} 3$ & $\mathrm{HYM}$ & $\Longleftrightarrow$ & $\mathbb{A}: 3 \hookrightarrow 4$ & $\widetilde{\mathrm{CY}} 3$ & $\widetilde{\mathrm{HYM}}$ \\
\hline
\end{tabular}

Figure 1: Mirror symmetry.

algebra $\mathbb{C l}(d)$ corresponds to the Hodge-dual operator * : $\Omega^{k}(M) \rightarrow \Omega^{d-k}(M)$ in the exterior algebra $\Lambda^{*} M$ where $\Gamma^{A}(A=1, \ldots, d)$ are $d$-dimensional Dirac matrices obeying the Dirac algebra

$$
\left\{\Gamma^{A}, \Gamma^{B}\right\}=2 \delta^{A B} \mathbf{I}_{2^{[d / 2]}} .
$$

It is amusing to note that the Clifford algebra from a modern viewpoint can be thought of as a quantization of the exterior algebra [10], in the same way that the Weyl algebra is a quantization of the symmetric algebra. In particular, the Clifford map (4) implies that the Lorentz generators $J^{A B} \equiv$ $(1 / 4)\left[\Gamma^{A}, \Gamma^{B}\right]$ in $\mathbb{C l}(d)$ are in one-to-one correspondence with two forms in the space $\Omega^{2}(M)$. And the representation space of the Clifford algebra is a spinor vector space whose elements are called fermions and essential ingredients in Standard Model. It may also be worthwhile to remark that any physical force is represented by two forms in the exterior algebra taking values in a classical Lie algebra. In addition recall that the representation of Clifford algebra in even dimensions is reducible and its irreducible representations are given by chiral fermions. Then the isomorphism (C) implies that there must be a corresponding irreducible decomposition of two forms in $\Lambda^{*} M$. This fact, in our case, has a nontrivial consequence for the Riemann curvature tensors $R_{A B}=$ $(1 / 2) R_{A B C D} e^{C} \wedge e^{D}$ since the $\operatorname{spin}(d)$ Lie algebra indices $(A, B)$ and the form indices $(C, D)$ must have an identical structure in a representation space of the Lorentz symmetry according to the isomorphism (C). Our principal concern is then to pin down a geometrical consequence of the rudimentary fact (A) after implementing the isomorphisms (B) and (C) to sixdimensional CY manifolds.

Let us briefly state the result summarized in the Figure 1 in advance. Compared to the four-dimensional case [11-14], some acute changes arise. First of all, there are two sources of two forms on an orientable six-dimensional manifold $M$. One is of course usual two forms in $\Omega^{2}(M)$ and the other is the Hodge duality of four forms in $\Omega^{4}(M)$. Therefore, the vector space of two forms is doubled in six dimensions:

$$
\Lambda^{2}(M) \equiv \Omega^{2}(M) \oplus * \Omega^{4}(M) .
$$

The doubling of two forms is resonant with the fact that the irreducible representation of Lorentz symmetry is given by the chiral Lorentz generators $J_{+}^{A B} \equiv(1 / 2)\left(\mathbf{I}_{8} \pm \Gamma_{7}\right) J^{A B}$. Definitely it corresponds to the mixture of two forms and four forms in $\Lambda^{2}(M)$ according to the correspondence $\left(\Gamma_{7} \leftrightarrow *\right)$. Since we need to take an irreducible representation of Lorentz symmetry, this demands us to think of the irreducible components of Riemann curvature tensors as a sum of the usual curvature tensors $R_{A B}$ and dual curvature tensors defined by
$\widetilde{R}_{A B} \equiv(* G)_{A B}=d \widetilde{\omega}_{A B}+\widetilde{\omega}_{A C} \wedge \widetilde{\omega}_{C B}$, where $G_{A B}$ is a 4 -form tensor taking values in $\operatorname{spin}(6) \cong s u(4)$ Lie algebra [14]. Moreover, it is necessary to impose the torsion-free condition for both spin connections, $\omega_{A B}$ and $\widetilde{\omega}_{A B}$, which leads to the symmetry property of the curvature tensors: $R_{C D A B}=R_{A B C D}$ and $\widetilde{R}_{C D A B}=\widetilde{R}_{A B C D}$. This is another reason why two kinds of indices $([A B],[C D])$ must be treated symmetrically although they belong to different vector spaces. To summarize, the Hodge duality admits two independent types of curvature tensors $\left(R_{A B C D} \oplus \widetilde{R}_{A B C D}\right)$ and they have to be decomposed according to the irreducible representation of $\operatorname{spin}(6) \cong s u(4)$ Lie algebra. In the end, the duplication of curvature tensors leads to the doubling for the variety of six-dimensional spin manifolds.

It might be stressed that the doubling of six-dimensional spin manifolds is an inevitable consequence of the elementary facts $(A, B, C)$. It should be instructive to apply the foregoing propositions $(\mathrm{A}, \mathrm{B}, \mathrm{C})$ to four manifolds to grasp their significance [12-14] although the four-dimensional situation is in stark contrast to the six-dimensional case. In four dimensions, the Lorentz group $\operatorname{Spin}(4)$ is isomorphic to $S U(2)_{L} \times$ $S U(2)_{R}$ whose Lie algebras $s u(2)_{L, R}$ consist of chiral Lorentz generators $J_{ \pm}^{A B} \equiv \Gamma_{ \pm} J^{A B}$ with $\Gamma_{ \pm}=(1 / 2)\left(\mathbf{I}_{4} \pm \Gamma_{5}\right)$ for chiral and antichiral representations. The splitting of the Lie algebra, $\operatorname{spin}(4) \cong s u(2)_{L} \oplus s u(2)_{R}$, is precisely isomorphic to the canonical decomposition of the vector space $\Omega^{2}(M)$ of two forms:

$$
\Omega^{2}(M)=\Omega_{+}^{2}(M) \oplus \Omega_{-}^{2}(M),
$$

where $\Omega_{ \pm}^{2}(M) \equiv P_{ \pm} \Omega^{2}(M)$ and $P_{ \pm}=(1 / 2)(1 \pm *)$. That is, the six-dimensional vector space $\Omega^{2}(M)$ of two forms splits canonically into the sum of three-dimensional vector spaces of self-dual and anti-self-dual two forms. One can apply the canonical splitting of the two vector spaces to Riemann curvature tensors simultaneously according to (4). It results in the well-known decomposition of the curvature tensor $R$ into irreducible components $[15,16]$, schematically given by

$$
R=\left(\begin{array}{cc}
W^{+}+\frac{1}{12} s & B \\
B^{T} & W^{-}+\frac{1}{12} s
\end{array}\right),
$$

where $s$ is the scalar curvature, $B$ is the traceless Ricci tensor, and $W^{ \pm}$are the (anti-)self-dual Weyl tensors. An important lesson from the four-dimensional example is that the irreducible (chiral) representation of Lorentz symmetry corresponds to the canonical split (7) of two forms with the projection operator $P_{ \pm}=(1 / 2)(1 \pm *)$. We observe that the same analysis in six dimensions brings about a more dramatic 
result due to the fact that $6=2+4$. The doubling of sixdimensional spin manifolds will be important to understand why CY manifolds arise with a mirror pair.

The gauge theory formulation of six-dimensional spin manifolds also leads to a valuable perspective for the doubling. The first useful access is to identify a gauge theory object corresponding to a CY 3-fold in the same sense that a gravitational instanton (or a hyper-Kähler manifold) can be identified with an $S U$ (2) Yang-Mills instanton in four dimensions [11-13]. An obvious guess goes toward a sixdimensional generalization of the four-dimensional YangMills instantons known as Hermitian Yang-Mills (HYM) instantons. Indeed this relationship has been well-known to string theorists and mathematicians under the name of the Donaldson-Uhlenbeck-Yau (DUY) theorem $[17,18]$. We quote a paragraph in [19] (Page 221) to clearly summarize this picture.

The point of intersection between the Calabi conjecture and the DUY theorem is the tangent bundle. And here's why: once you have proved the existence of CY manifolds, you have not only those manifolds but also their tangent bundles, because every manifold has one. Since the tangent bundle is defined by the CY manifold, it inherits its metric from the parent manifold (in this case, the CY). The metric for the tangent bundle, in other words, must satisfy the CY equations. It turns out, however, that, for the tangent bundle, the Hermitian Yang-Mills equations are the same as the CY equations, provided the background metric you have selected is the CY. Consequently, the tangent bundle, by virtue of satisfying the CY equations, automatically satisfies the Hermitian Yang-Mills equations, too.

If a CY manifold $M$ can be related to a HYM instanton, a natural question immediately arises. Since a CY manifold $M$ has a mirror manifold, there will be a mirror CY manifold $\widetilde{M}$ obeying the mirror relation (1). This in turn implies that there must be a mirror HYM instanton which can be derived from the mirror CY manifold $\widetilde{M}$. Thus we want to understand the relation between the HYM instanton and its mirror instanton from the gauge theory perspective. Since the Lorentz group $\operatorname{Spin}(6)$ is isomorphic to $S U(4)$, the chiral and antichiral representations 4 and $4^{\prime}$ of $\operatorname{Spin}(6)$ are equivalent to the fundamental and antifundamental representations 4 and $\overline{4}$ of $S U(4)$. Recall that the fundamental representation 4 of $S U(4)$ is a complex representation and so its complex conjugate $\overline{4}$ is an inequivalent representation different from 4 . Therefore, given a CY manifold $M$, one can embed the HYM instanton inherited from $M$ into two different representations. But this situation is equally true for the mirror CY manifold $\widetilde{M}$. Thus there is a similar doubling for the variety of HYM instantons as occurred to CY manifolds, as summarized in Figure 1.

It may be interesting to compare this situation with the four-dimensional case $[13,14]$. In four dimensions, the positive and negative chirality spinors of $\operatorname{Spin}(4)$ are given by $S U(2)_{L}$ and $S U(2)_{R}$ spinors, $2_{L}$ and $2_{R}$, respectively. In this case, it is necessary to have two independent $S U(2)$ factors to be compatible with the splitting (7) because the irreducible representation of $S U(2)$ is real. It is interesting to see how (A, B, C) take part in the conspiracy. First, a CY 2-fold can be mapped to a self-dual or $S U(2)_{L}$ instanton which lives in the chiral representation $\mathbf{2}_{L}$, while a mirror CY 2-fold is isomorphically related to an anti-self-dual or $S U(2)_{R}$ instanton in the antichiral representation $2_{R}$. For this correspondence, the $S U(2)$ gauge group of Yang-Mills instantons is identified with the holonomy group of CY 2fold. This picture is generalized to six dimensions in an interesting way. In six dimensions, the canonical splitting (7) is applied to the enlarged vector space (6) as

$$
\Lambda^{2}(M)=\Omega_{+}^{2}(M) \oplus \Omega_{-}^{2}(M),
$$

where the decomposition $\Omega_{ \pm}^{2}(M)$ is dictated by the chiral splitting $J^{A B}=J_{+}^{A B} \oplus J_{-}^{A B}$ according to the isomorphism (C). From the gauge theory perspective, the splitting (9) is also compatible with the fundamental and antifundamental representations of the gauge group $S U(4) \cong \operatorname{Spin}(6)$ because the chiral representation of $\operatorname{Spin}(6)$ is identified with the fundamental representation of SU(4). After all, we will get the picture that the HYM instanton on TM embedded in the fundamental representation $\mathbf{4}$ is mirror to the HYM instanton on $T \widetilde{M}$ in the antifundamental representation $\overline{4}$. This structure is summed up in Figure 1, where CY3 refers to a CY 3 -fold $M$ and $\widetilde{\mathrm{CY} 3}$ its mirror $\widetilde{M}$. And HYM denotes a HYM instanton on $M$ in the complex representation either 3 or $\overline{3}$ of $S U(3) \subset S U(4)$ and $\widetilde{\mathrm{HYM}}$ its mirror on $\widetilde{M}$ in the opposite complex representation.

The purpose of this paper is to understand the structure in Figure 1. To the best of our knowledge, there is no concrete work to address the mirror symmetry based on the picture in Figure 1 although the mirror symmetry has been extensively studied so far. We will show that CY manifolds and HYM instantons exist with mirror pairs as a consequence of the doubling (6) of two forms in six dimensions. It is arguably a remarkable consequence of the mysterious Clifford isomorphism (C).

This paper is organized as follows. In Section 2, we formulate $d$-dimensional Euclidean gravity as a Spin $(d)$ Yang-Mills gauge theory. The explicit relations between gravity and gauge theory variables are established. In particular, we construct the dual curvature tensors $\widetilde{R}_{A B} \equiv(* G)_{A B}=d \widetilde{\omega}_{A B}+\widetilde{\omega}_{A C} \wedge \widetilde{\omega}_{C B}$ that are necessary for an irreducible representation of Lorentz symmetry. We observe that the geometric structure described by dual spin connections $\widetilde{\omega}_{A B}$ and curvature tensors $\widetilde{R}_{A B}$ is exactly parallel to the usual one described by $\left(\omega_{A B}, R_{A B}\right)$ and so clarify why the variety of orientable spin manifolds is doubled.

We apply in Section 3 the gauge theory formulation to six-dimensional Riemannian manifolds. For that purpose we devise a six-dimensional version of the 't Hooft symbols which realizes the isomorphism between spin(6) Lorentz algebra and $s u(4)$ Lie algebra. As the spin(6) Lorentz algebra has two irreducible spinor representations, there are accordingly two kinds of the 't Hooft symbols depending 
on the chirality of irreducible spin(6) representations. Our construction of six-dimensional 't Hooft symbols is new to the best of our knowledge. Using this construction, we impose the Kähler condition on the 't Hooft symbols. This is done by projecting the 't Hooft symbols to $U(3)$-valued ones and so results in the reduction of the gauge group from $S U(4)$ to $U(3)$. After imposing the Ricci-flat condition, the gauge group in Yang-Mills gauge theory is further reduced to $S U$ (3). This result is utilized to show that six-dimensional CY manifolds can be recast as HYM instantons in SU(3) YangMills gauge theory. We elucidate why the canonical splitting (9) of six-dimensional spin manifolds corresponds to the chiral representation of Spin(6). It turns out that this splitting is equally applied to CY manifolds as well as HYM instantons.

In Section 4, we apply the results in Section 3 to CY manifolds to see how the mirror symmetry between them can be explained by the doubling of six-dimensional spin manifolds. We observe that it is always possible to find a pair of CY manifolds such that their Euler characteristics in different chiral representations obey the mirror relation (1). This implies that a pair of CY manifolds in the opposite chiral representation are mirror to each other as indicated by the arrow $(\Leftrightarrow)$ in Figure 1 .

In Section 5, we revisit the relation between CY manifolds and HYM instantons to discuss the mirror symmetry from a completely gauge theory perspective. We show that a pair of HYM instantons embedded in different complex representations $\mathbf{4}$ and $\overline{4}$ correspond to a mirror pair of $\mathrm{CY}$ manifolds as summarized in Figure 1. This result is consistent with the mirror symmetry because the integral of the third Chern class $c_{3}(E)$ for a vector bundle $E$ is equal to the Euler characteristic of tangent bundle $T M$ when $E=T M$ and the third Chern class has a desired sign flip between a complex vector bundle $E$ in the fundamental representation and its conjugate bundle $\bar{E}$ in the antifundamental representation. Therefore, we confirm the picture in Figure 1 that the mirror symmetry between CY manifolds can be understood as a mirror pair of HYM instantons in holomorphic vector bundles.

Finally we recapitulate in Section 6 the results obtained in this paper and conclude the paper with a few speculative remarks.

In Appendix A, we fix the basis for the chiral representation of Spin(6) and the fundamental representation of $S U(4)$ and list their structure constants. In Appendix B, we present an explicit construction of the six-dimensional 't Hooft symbols and their algebraic properties in each chiral basis.

\section{Gravity as a Gauge Theory}

In this section we consider the gauge theory formulation of Riemannian manifolds taking values in an irreducible spinor representation of the Lorentz group $[13,14]$. This section is to establish the notation for the doubled variety of Riemannian manifolds, but more detailed exposition will be deferred to the next section. On a Riemannian manifold $M$ of dimension $d$, the spin connection is a $\operatorname{spin}(d)$-valued one form and can be identified, in general, with a $\operatorname{Spin}(d)$ gauge field. In order to make an explicit identification between the spin connections and the corresponding gauge fields, let us first consider the $d$-dimensional Dirac algebra (5) where $\Gamma^{A}(A=1, \ldots, d)$ are Dirac matrices. Then the $\operatorname{spin}(d)$ Lorentz generators are given by

$$
J^{A B}=\frac{1}{4}\left[\Gamma^{A}, \Gamma^{B}\right]
$$

which satisfy the following Lorentz algebra:

$$
\begin{aligned}
& {\left[J^{A B}, J^{C D}\right]} \\
& \quad=-\left(\delta^{A C} J^{B D}-\delta^{A D} J^{B C}-\delta^{B C} J^{A D}+\delta^{B D} J^{A C}\right) .
\end{aligned}
$$

The spin connection is defined by $\omega=(1 / 2) \omega_{A B} J^{A B}$, which transforms in the standard way as a $\operatorname{Spin}(d)$ gauge field under local Lorentz transformations

$$
\omega \longrightarrow \omega^{\prime}=\Lambda \omega \Lambda^{-1}+\Lambda d \Lambda^{-1},
$$

where $\Lambda=e^{(1 / 2) \lambda_{A B}(x) J^{A B}} \in \operatorname{Spin}(d)$.

In even dimensions, the spinor representation is reducible and its irreducible representations are given by positive and negative chiral representations. In next section we will provide an explicit chiral representation for the six-dimensional case. The Lorentz generators for the chiral representation are given by

$$
J^{A B}=\left(\begin{array}{cc}
J_{+}^{A B} & 0 \\
0 & J_{-}^{A B}
\end{array}\right),
$$

where $J_{ \pm}^{A B}=\Gamma_{ \pm} J^{A B}$ and $\Gamma_{ \pm}=(1 / 2)\left(\mathbf{I}_{2^{[d / 2]}} \pm \Gamma_{d+1}\right)$. Therefore, the spin connection in the chiral representation takes the form

$$
\begin{aligned}
& \varpi=\frac{1}{2}{\omega_{A B}}^{A B}=\left(\begin{array}{cc}
\omega^{(+)} & 0 \\
0 & \omega^{(-)}
\end{array}\right) \\
& =\frac{1}{2}\left(\begin{array}{cc}
\omega_{A B}^{(+)} J_{+}^{A B} & 0 \\
0 & \omega_{A B}^{(-)} J_{-}^{A B}
\end{array}\right) .
\end{aligned}
$$

Here we used a sloppy notation for $\omega$ which must be understood as $\boldsymbol{\omega}=(1 / 2) \omega_{\mathrm{AB}} J^{\mathbf{A B}}$, where $\mathbf{A}=\left(A, A^{\prime}\right), \mathbf{B}=$ $\left(B, B^{\prime}\right)$ and $\omega_{A B^{\prime}}=\omega_{A^{\prime} B}=0$. For a notational simplicity we will use this notation since it will not introduce too much confusion. Note that the spin connections $\omega^{(+)}$and $\omega^{(-)}$are considered as independent since they resulted from the doubling of one form due to the Hodge duality, as will be shown later.

Now we introduce a $\operatorname{Spin}(d)$ gauge field defined by

$$
\begin{gathered}
\mathbb{A}=A^{a} \mathbb{T}^{a}=\left(\begin{array}{cc}
A^{(+) a} T^{a} & 0 \\
0 & A^{(-) a}\left(T^{a}\right)^{*}
\end{array}\right), \\
\mathbb{T}^{a}=\left(\begin{array}{cc}
T^{a} & 0 \\
0 & \left(T^{a}\right)^{*}
\end{array}\right) \in \operatorname{spin}(d),
\end{gathered}
$$


where $A^{( \pm) a}=A_{M}^{( \pm) a} d x^{M}(a=1, \ldots, d(d-1) / 2)$ are one-form connections on $M$. We will take definition (15) by adopting the group isomorphism (3). The Lie algebra generators are matrices obeying the commutation relation

$$
\left[\mathbb{T}^{a}, \mathbb{T}^{b}\right]=-f^{a b c} \mathbb{T}^{c}
$$

where $T^{a}$ and $\left(T^{a}\right)^{*}$ are generators in a representation $R$ and its conjugate representation $\bar{R}$, respectively. The identification we want to make is then given by

$$
\omega=\frac{1}{2} \omega_{A B} J^{A B} \cong \mathbb{A}=A^{a} \mathbb{T}^{a} .
$$

Then the Lorentz transformation (12) can be interpreted as a usual gauge transformation

$$
\mathbb{A} \longrightarrow \mathbb{A}^{\prime}=\Lambda \mathbb{A} \Lambda^{-1}+\Lambda d \Lambda^{-1}
$$

where $\Lambda=e^{\lambda^{a}(x) \mathbb{U}^{a}} \in \operatorname{Spin}(d)$. The Riemann curvature tensor is defined by [8]

$$
\begin{aligned}
\mathscr{R} & =\frac{1}{2} \mathscr{R}_{A B} J^{A B}=d \omega+\omega \wedge \omega \\
& =\frac{1}{2}\left(d \omega_{A B}+\omega_{A C} \wedge \omega_{C B}\right) J^{A B} \\
& =\frac{1}{2}\left(\begin{array}{cc}
R_{A B}^{(+)} J_{+}^{A B} & 0 \\
0 & R_{A B}^{(-)} J_{-}^{A B}
\end{array}\right)
\end{aligned}
$$

where $R_{A B}^{( \pm)}=(1 / 2)\left(\partial_{M} \omega_{N A B}^{( \pm)}-\partial_{N} \omega_{M A B}^{( \pm)}+\omega_{M A C}^{( \pm)} \omega_{N C B}^{( \pm)}-\right.$ $\left.\omega_{N A C}^{( \pm)} \omega_{M C B}^{( \pm)}\right) d x^{M} \wedge d x^{N}$. Or, in terms of gauge theory variables, it is given by

$$
\begin{aligned}
\mathbb{F} & =F^{a} \mathbb{T}^{a}=d \mathbb{A}+\mathbb{A} \wedge \mathbb{A} \\
& =\left(d A^{a}-\frac{1}{2} f_{b c}{ }^{a} A^{b} \wedge A^{c}\right) \mathbb{T}^{a} \\
& =\frac{1}{2}\left(\begin{array}{cc}
F^{(+) a} T^{a} & 0 \\
0 & F^{(-) a}\left(T^{a}\right)^{*}
\end{array}\right),
\end{aligned}
$$

where $F^{( \pm) a}=(1 / 2)\left(\partial_{M} A_{N}^{( \pm) a}-\partial_{N} A_{M}^{( \pm) a}-f^{a b c} A_{M}^{( \pm) b} A_{N}^{( \pm) c}\right) d x^{M} \wedge$ $d x^{N}$.

As we outlined in Section 1, in addition to the usual curvature tensor $R_{A B}=d \omega_{A B}+\omega_{A C} \wedge \omega_{C B}$, we need to introduce the dual curvature tensor defined by

$$
\widetilde{R}_{A B} \equiv(* G)_{A B}=d \widetilde{\omega}_{A B}+\widetilde{\omega}_{A C} \wedge \widetilde{\omega}_{C B},
$$

where $G_{A B}$ is a $(d-2)$-form tensor taking values in $\operatorname{spin}(d)$ Lie algebra. One may consider the dual spin connection $\widetilde{\omega}_{A B} \equiv(* \theta)_{A B}$ as the Hodge dual of a $(d-1)$-form $\theta_{A B}$ in $\operatorname{spin}(d)$ Lie algebra. It is useful to introduce the adjoint exterior differential operator $\delta: \Omega^{k}(M) \rightarrow \Omega^{k-1}(M)$ defined by

$$
\delta=(-1)^{d k+d+1} * d *
$$

where the Hodge-dual operator $*: \Omega^{k}(M) \rightarrow \Omega^{d-k}(M)$ obeys the well-known relation

$$
*^{2} \alpha=(-1)^{k(d-k)} \alpha
$$

for $\alpha \in \Omega^{k}(M)$. Using the adjoint differential operator $\delta$, the $\operatorname{spin}(d)$-valued $(d-2)$-form $G_{A B}$ in (21) can be written as

$$
G_{A B}=(-)^{d-1} \delta \theta_{A B}+\theta_{A C} \bar{\wedge} \theta_{C B},
$$

where we devised a simplifying notation

$$
\alpha \bar{\wedge} \beta \equiv *((* \alpha) \wedge(* \beta)) \in \Omega^{p+q-d}(M)
$$

for $\alpha \in \Omega^{p}(M)$ and $\beta \in \Omega^{q}(M)$. Using the nilpotency of the adjoint differential operator $\delta$, that is $\delta^{2}=0$, one can derive the (second) Bianchi identity:

$$
\delta G_{A B}+(-)^{d-1}\left(\theta_{A C} \bar{\wedge} G_{C B}-G_{A C} \bar{\wedge} \theta_{C B}\right)=0 .
$$

It may be compared with the ordinary Bianchi identity in general relativity written as

$$
d R_{A B}+\omega_{A C} \wedge R_{C B}-R_{A C} \wedge \omega_{C B}=0 .
$$

Let us also introduce dual vielbeins $\widetilde{e}^{A} \equiv(* h)^{A}$, where $h^{A} \in \Omega^{d-1}(M)$, in addition to the usual vielbeins $e^{A}(A=$ $1, \ldots, d)$ which independently form a local orthonormal coframe at each spacetime point in $M$. We combine the dual one-form $\widetilde{e}^{A}$ with the usual coframe $e^{A}$ to define a matrix of vielbeins:

$$
\mathfrak{E}=\mathfrak{E}_{A} \Gamma^{A}=\left(\begin{array}{cc}
0 & e^{(+) A} \gamma^{A} \\
e^{(-) A} \bar{\gamma}^{A} & 0
\end{array}\right),
$$

where

$$
e^{( \pm) A} \equiv \frac{1}{2}\left(e^{A} \pm \widetilde{e}^{A}\right) .
$$

The coframe basis $\left\{e^{( \pm) A} \in \Gamma\left(T^{*} M\right)\right\}$ defines dual vectors $E_{A}^{( \pm)}=E_{A}^{( \pm) M} \partial_{M} \in \Gamma(T M)$ by a natural pairing:

$$
\left\langle e^{( \pm) A}, E_{B}^{( \pm)}\right\rangle=\delta_{B}^{A} .
$$

The above pairing leads to the relation $e_{M}^{( \pm) A} E_{B}^{( \pm) M}=\delta_{B}^{A}$. Since we regard the spin connections $\omega^{(+)}$and $\omega^{(-)}$as independent, let us consider two kinds of geometrical data on a spin manifold $M$, dubbed $\mathbb{A}$ and $\mathbb{B}$ classes:

$$
\begin{aligned}
& \mathbb{A}:\left(e^{(+) A}, \omega_{A B}^{(+)}\right), \\
& \mathbb{B}:\left(e^{(-) A}, \omega_{A B}^{(-)}\right) .
\end{aligned}
$$

We emphasize that the geometric structure of a $d$ dimensional spin manifold can be described by either type $A$ or type $\mathbb{B}$ but they should be regarded as independent even topologically. In other words, we can separately consider a Riemannian metric for each class given by

$$
\begin{aligned}
d s_{ \pm}^{2} & =\delta_{A B} e^{( \pm) A} \otimes e^{( \pm) B}=\delta_{A B} e_{M}^{( \pm) A} e_{N}^{( \pm) B} d x^{M} \otimes d x^{N} \\
& \equiv g_{M N}^{( \pm)}(x) d x^{M} \otimes d x^{N}
\end{aligned}
$$


or

$$
\begin{aligned}
\left(\frac{\partial}{\partial s}\right)_{ \pm}^{2} & =\delta^{A B} E_{A}^{( \pm)} \otimes E_{B}^{( \pm)}=\delta^{A B} E_{A}^{( \pm) M} E_{B}^{( \pm) N} \partial_{M} \otimes \partial_{N} \\
& \equiv g_{( \pm)}^{M N}(x) \partial_{M} \otimes \partial_{N} .
\end{aligned}
$$

In order to recover general relativity from the gauge theory formulation of gravity, it is necessary to impose the torsion-free condition; that is,

$$
T^{( \pm) A}=d e^{( \pm) A}+\omega_{B}^{( \pm) A} \wedge e^{( \pm) B}=0 .
$$

As a result, the spin connections are determined by vielbeins, that is, $\omega^{( \pm)}=\omega^{( \pm)}\left(e^{( \pm)}\right)$, from which one can deduce the first Bianchi identity

$$
R_{A B}^{( \pm)} \wedge e^{( \pm) B}=0
$$

where the curvature tensors $R_{A B}^{( \pm)}$are defined by (19). It is not difficult to see that (35) leads to the symmetry property for the Riemann curvature tensors $R_{A B}^{( \pm)} \equiv(1 / 2) R_{A B C D}^{( \pm)} e^{( \pm) C} \wedge e^{( \pm) D}$; $R_{A B C D}^{( \pm)}=R_{C D A B}^{( \pm)}$. It may be convenient to introduce the torsion matrix $\mathbb{T}$ defined by

$$
\mathbb{T}=d \mathfrak{E}+\omega \wedge \mathfrak{F}+\mathfrak{E} \wedge \widehat{\widehat{\omega}}=\left(\begin{array}{cc}
0 & T^{(+) A} \gamma^{A} \\
T^{(-) A} \bar{\gamma}^{A} & 0
\end{array}\right),
$$

where we have defined the inverted spin connection $\widehat{\omega} \equiv$ $(1 / 2) \widehat{\omega}_{A B} \widetilde{J}^{A B}=(1 / 2)\left(\begin{array}{cc}\omega_{A B}^{(-)} J_{-}^{A B} & 0 \\ 0 & \omega_{A B}^{(+)} J_{+}^{A B}\end{array}\right)$. It is straightforward to show that

$$
d \mathbb{T}=\frac{1}{2}\left(\begin{array}{cc}
0 & R_{A B}^{(+)} \wedge e^{(+) B} \gamma^{A} \\
R_{A B}^{(-)} \wedge e^{(-) B} \bar{\gamma}^{A} & 0
\end{array}\right)
$$

and so the first Bianchi identity (35) is automatic because of the torsion-free condition, $\mathbb{T}=0$. Similarly, using definition (19), it is easy to derive the second Bianchi identity, $\mathscr{D} \mathscr{R} \equiv$ $d \mathscr{R}+\omega \wedge \mathscr{R}-\mathscr{R} \wedge \omega=0$, whose matrix form reads as

$$
\mathscr{D} \mathscr{R}=\left(\begin{array}{cc}
D^{(+)} R^{(+)} & 0 \\
0 & D^{(-)} R^{(-)}
\end{array}\right)=0,
$$

where

$$
D^{( \pm)} R^{( \pm)} \equiv d R^{( \pm)}+\omega^{( \pm)} \wedge R^{( \pm)}-R^{( \pm)} \wedge \omega^{( \pm)} .
$$

In terms of gauge theory variables, it can be stated as $\mathbb{D F} \equiv$ $d \mathbb{F}+\mathbb{A} \wedge \mathbb{F}-\mathbb{F} \wedge \mathbb{A}=0$ or $D^{( \pm)} F^{( \pm)} \equiv d F^{( \pm)}+A^{( \pm)} \wedge F^{( \pm)}-$ $F^{( \pm)} \wedge A^{( \pm)}=0$.

To sum up, a $d$-dimensional orientable Riemannian manifold admits a globally defined volume form which leads to the isomorphism between $\Omega^{k}(M)$ and $\Omega^{d-k}(M)$. In particular, it doubles the two-form vector space which leads to the enlargement for the geometric structure of Riemannian manifolds. The Hodge duality $*: \Omega^{k}(M) \rightarrow \Omega^{d-k}(M)$ is thus the origin of the doubling for the variety of Riemannian manifolds. One is described by $\left(e^{A}, \omega_{A B}, R_{A B}\right)$ and the other independent construction is given by $\left(\widetilde{e}^{A}, \widetilde{\omega}_{A B}, \widetilde{R}_{A B}\right) \cong$ $*\left(h^{A}, \theta_{A B}, G_{A B}\right)$. According to the isomorphism (C), they are decomposed into two irreducible representations of Lorentz symmetry. In the next section we will apply the irreducible decomposition to six-dimensional spin manifolds to see why the variety of Riemannian manifolds is doubled.

\section{Spinor Representation of Six-Dimensional Riemannian Manifolds}

We will apply the gauge theory formulation in the previous section to six-dimensional Riemannian manifolds. For this purpose, the Spin(6) Lorentz group for Euclidean gravity will be identified with the $S U$ (4) gauge group in Yang-Mills gauge theory. A motivation for the gauge theory formulation of sixdimensional Euclidean gravity is to identify a gauge theory object corresponding to a CY manifold and to understand the mirror symmetry of CY manifolds in terms of Yang-Mills gauge theory. Because our gauge theory formulation is based on identification (17), we will restrict ourselves to orientable six-dimensional manifolds with spin structure and consider a spinor representation of $\operatorname{Spin}(6)$ in order to scrutinize the relationship.

Let us start with the Clifford algebra $\mathbb{C l ( 6 )}$ whose generators are given by

$$
\mathbb{C l}(6)=\left\{\mathbf{I}_{8}, \Gamma^{A}, \Gamma^{A B}, \Gamma^{A B C}, \Gamma_{7} \Gamma^{A B}, \Gamma_{7} \Gamma^{A}, \Gamma_{7}\right\},
$$

where $\Gamma^{A}(A=1, \ldots, 6)$ are six-dimensional Dirac matrices satisfying the algebra (5) and $\Gamma^{A_{1} A_{2} \cdots A_{k}} \equiv(1 / k !) \Gamma^{\left[A_{1}\right.} \Gamma^{A_{2}} \cdots \Gamma^{\left.A_{k}\right]}$ assumes the complete antisymmetrization of indices. $\Gamma_{7} \equiv$ $i \Gamma^{1} \cdots \Gamma^{6}$ is the chiral matrix given by (A.6). According to the isomorphism (4), the Clifford algebra (40) can be isomorphically mapped to the exterior algebra of a cotangent bundle $T^{*} M$

$$
\mathbb{C l}(6) \cong \Lambda^{*} M=\bigoplus_{k=0}^{6} \Omega^{k}(M),
$$

where the chirality operator $\Gamma_{7}$ corresponds to the Hodgedual operator $*: \Omega^{k}(M) \rightarrow \Omega^{6-k}(M)$.

The spinor representation of $\operatorname{Spin}(6)$ can be constructed by 3 fermion creation operators $a_{i}^{*}(i=1,2,3)$ and the corresponding annihilation operators $a^{j}(j=1,2,3)$ (see Appendix 5.A in [1]). This fermionic system can be represented in a Hilbert space $V$ of dimension 8 with a Fock vacuum $|\Omega\rangle$, annihilated by all the annihilation operators. The states in $V$ are obtained by acting the product of $k$ creation operators $a_{i_{1}}^{*} \cdots a_{i_{k}}^{*}$ on the vacuum $|\Omega\rangle$; that is,

$$
V=\bigoplus_{k=0}^{3}\left|\Omega_{i_{1} \cdots i_{k}}\right\rangle=\bigoplus_{k=0}^{3} a_{i_{1}}^{*} \cdots a_{i_{k}}^{*}|\Omega\rangle .
$$

The spinor representation of the algebra (40) is reducible and has two irreducible spinor representations. Indeed the Hilbert space $V$ splits into the spinors $S_{ \pm}$of positive and negative chirality; that is, $V=S_{+} \oplus S_{-}$, each of dimension 4 . If the 
Fock vacuum $|\Omega\rangle$ has positive chirality, the positive chirality spinors of $\operatorname{Spin}(6)$ are states given by

$$
S_{+}=\bigoplus_{k \text { even }}\left|\Omega_{i_{1} \cdots i_{k}}\right\rangle=|\Omega\rangle+\left|\Omega_{i j}\right\rangle \equiv \mathbf{4}
$$

while the negative chirality spinors are those obtained by

$$
S_{-}=\bigoplus_{k \text { odd }}\left|\Omega_{i_{1} \cdots i_{k}}\right\rangle=\left|\Omega_{i}\right\rangle+\left|\Omega_{i j k}\right\rangle \equiv \overline{\mathbf{4}}
$$

As spin(6) Lorentz algebra is isomorphic to $s u(4)$ Lie algebra, the positive and negative chirality spinors of $\operatorname{spin}(6)$ can be identified with the fundamental representation $\mathbf{4}$ and the antifundamental representation $\overline{4}$ of $s u(4)$, respectively [1]. As a result, the chiral spinor representations $S_{+}$and $S_{-}$of Spin(6) are identified with the fundamental representations 4 and $\overline{4}$ of $S U(4)$.

One can form a direct product of the fundamental representations $\mathbf{4}$ and $\overline{\mathbf{4}}$ in order to classify the Clifford generators in (40):

$$
\begin{aligned}
& \mathbf{4} \otimes \overline{\mathbf{4}}=\mathbf{1} \oplus \mathbf{1 5}=\left\{\Gamma_{+}, \Gamma_{+}^{A B}\right\}, \\
& \overline{\mathbf{4}} \otimes \mathbf{4}=\mathbf{1} \oplus \mathbf{1 5}=\left\{\Gamma_{-}, \Gamma_{-}^{A B}\right\}, \\
& \mathbf{4} \otimes \mathbf{4}=\mathbf{6} \oplus \mathbf{1 0}=\left\{\Gamma_{+}^{A}, \Gamma_{+}^{A B C}\right\}, \\
& \overline{\mathbf{4}} \otimes \overline{\mathbf{4}}=\mathbf{6} \oplus \mathbf{1 0}=\left\{\Gamma_{-}^{A}, \Gamma_{-}^{A B C}\right\},
\end{aligned}
$$

where $\Gamma_{ \pm} \equiv(1 / 2)\left(\mathbf{I}_{8} \pm \Gamma_{7}\right)$ are the projection operators onto the space of definite chirality and $\Gamma_{ \pm}^{A_{1} A_{2} \cdots A_{k}} \equiv \Gamma_{ \pm} \Gamma^{A_{1} A_{2} \cdots A_{k}}$. Note that 15 in (45) and (46) is the adjoint representation of $S U(4)$ and 6 and 10 in (47) and (48) are the antisymmetric and symmetric representations of $S U(4)$, respectively. See Appendix A for the Lie algebra generators in the chiral representation of $\operatorname{Spin}(6)$ and the fundamental representation of $S U$ (4). It is important to notice that $\Gamma_{+}^{A B} \in \mathbf{1 5}$ and $\Gamma_{-}^{A B} \in \mathbf{1 5}$ are independent of each other; that is, $\left[\Gamma_{+}^{A B}, \Gamma_{-}^{C D}\right]=0$, and this doubling of the Clifford basis is parallel to the doubling of two forms according to the Clifford isomorphism (41) as will be clarified below.

We want to find the irreducible decomposition of Riemann curvature tensors under the Lorentz symmetry as the six-dimensional version of (8). As we noticed before, there are two kinds of Lorentz generators given by the irreducible components $\Gamma_{ \pm}^{A B}=\Gamma_{ \pm} \Gamma^{A B}$ which correspond to the chiral and antichiral representations of Lorentz algebra $\operatorname{spin}(6) \cong s u(4)$. Recall that (19) takes the following split of curvature tensors $\mathscr{R}=(1 / 2) \mathscr{R}_{A B} e^{A} \wedge e^{B}$ :

$$
\mathscr{R}_{A B}=R_{A B}^{(+)} \oplus R_{A B}^{(-)}=\left(F_{A B}^{(+) a} T_{1}^{a} \oplus F_{A B}^{(-) a} T_{2}^{a}\right)=\mathbb{F}_{A B}
$$

where $R_{A B}^{( \pm)}=\Gamma_{ \pm} \mathscr{R}_{A B}$ and both $T_{1}^{a}$ and $T_{2}^{a}$ obey the $s u(4)$ Lie algebra defined by (16). The doubling of $s u(4)$ Lie algebra in four-dimensional representations $R_{1}$ and $R_{2}$ on the right-hand side was considered in parallel to the spinor representation on the left-hand side. Since the Lorentz generators $J_{ \pm}^{A B}=\Gamma_{ \pm} J^{A B}$ are in one-to-one correspondence with two forms in the vector space (9), we identify the following map:

$$
\begin{aligned}
& J_{+}^{A B} \longleftrightarrow F_{A B}^{(+)}, \\
& J_{-}^{A B} \longleftrightarrow F_{A B}^{(-)} .
\end{aligned}
$$

Since the role of the chiral operator $\Gamma_{7}$ is parallel with the Hodge-dual operator $*: \Omega^{k}(M) \rightarrow \Omega^{6-k}(M)$, the chiral Lorentz generators $J_{ \pm}^{A B}=(1 / 4)\left(\mathbf{I}_{8} \pm \Gamma_{7}\right) \Gamma^{A B}=(1 / 4)\left(\Gamma^{A B} \mp\right.$ $\left(i / 4\right.$ !) $\left.\varepsilon_{A B C D E F} \Gamma^{C D E F}\right)$ correspond to the canonical split of the enlarged vector space (6). Therefore, the two forms $F^{( \pm)}=$ $(1 / 2) F_{A B}^{( \pm)} e^{( \pm) A} \wedge e^{( \pm) B}$ in (49) must be understood as the element of the irreducible vector space in (9); that is,

$$
F^{( \pm)} \in \Omega_{ \pm}^{2}(M) \text {. }
$$

As a result, $\mathscr{R}_{A B}$ on the left-hand side of (49) has twice as many components as the usual Riemann curvature tensor.

Let us summarize the gauge theory formulation in Section 2. Suppose that $J_{*}^{A B}$ and $T_{*}^{a}$ are Lie algebra generators in an irreducible representation $R_{*}$ of $\operatorname{Spin}(6)$ and $S U(4)$, respectively. First consider an $S U(4)$ gauge field $B=B^{a} T_{*}^{a}=$ $* C$ in the representation $R_{*}$ obtained by taking the Hodge duality of a four-form $C=C^{a} T_{*}^{a}$ and make the following identification:

$$
\begin{gathered}
\widetilde{\omega}=\frac{1}{2} \widetilde{\omega}_{A B} J_{*}^{A B} \cong B=B^{a} T_{*}^{a}, \\
\theta=\frac{1}{2} \theta_{A B} J_{*}^{A B} \cong C=C^{a} T_{*}^{a},
\end{gathered}
$$

where $\widetilde{\omega}=(1 / 2) \widetilde{\omega}_{A B} J_{*}^{A B}$ is the dual spin connection and $\widetilde{\omega}=* \theta$. Then the dual curvature tensors (21) and (24) are, respectively, written as

$$
\begin{aligned}
& \widetilde{F}=d B+B \wedge B=* H, \\
& H=(-)^{d-1} \delta C+C \bar{\wedge} C,
\end{aligned}
$$

where $H$ is a four-form field strength whose Hodge duality is the field strength $\widetilde{F}$ in $S U(4)$ gauge theory. The nilpotency of exterior differentials, $d^{2}=\delta^{2}=0$, immediately leads to the Bianchi identity

$$
\begin{aligned}
d \widetilde{F}+B \wedge \widetilde{F}-\widetilde{F} \wedge B & =0 \Longleftrightarrow \\
\delta H+(-)^{d-1}(C \bar{\wedge} H-H \bar{\wedge} C) & =0 .
\end{aligned}
$$

Hence the geometric structure described by the dual variables $\left(\widetilde{\omega}_{A B}, \widetilde{R}_{A B}\right)$ will be exactly parallel to the usual one described by $\left(\omega_{A B}, R_{A B}\right)$.

Thus it is natural to put the two geometric structures on an equal footing. Moreover, the irreducible representation of the Clifford algebra $\mathbb{C l}(6)$ suggests that the curvature tensors in (51) are given by the combination

$$
F^{( \pm)}=\frac{1}{2}(F \pm \widetilde{F})=\frac{1}{2}(F \pm * H) .
$$


One may note that, on an orientable (spin) manifold, the duplication of curvature tensors always happens by the Hodge duality. The combination (55) can be understood as follows. One may regard the Riemann tensor $\mathscr{R}_{A B}=$ $(1 / 2) \mathscr{R}_{A B C D} J^{C D}$ as a linear operator acting on the Hilbert space $V$ in (42). As $\mathscr{R}_{A B}$ contains two gamma matrices, it does not change the chirality of the vector space $V$. Therefore, we can represent it in a subspace of definite chirality as either $R_{A B}^{(+)}: S_{+} \rightarrow S_{+}$or $R_{A B}^{(-)}: S_{-} \rightarrow S_{-}$. The former case $R_{A B}^{(+)}: S_{+} \rightarrow$ $S_{+}$takes values in $\mathbf{4} \otimes \overline{\mathbf{4}}$ in (45) with a singlet being removed while the latter case $R_{A B}^{(-)}: S_{-} \rightarrow S_{-}$takes values in $\overline{4} \otimes 4$ in (46) with no singlet. This implies two independent identifications defined by

$$
\begin{aligned}
& \mathbb{A}: \frac{1}{2} R_{A B C D}^{(+)} J_{+}^{C D} \equiv F_{A B}^{(+) a}\left(T^{a} \oplus \mathbf{0}\right), \\
& \mathbb{B}: \frac{1}{2} R_{A B C D}^{(-)} J_{-}^{C D} \equiv F_{A B}^{(-) a}\left(\mathbf{0} \oplus\left(T^{a}\right)^{*}\right),
\end{aligned}
$$

where class $\mathbb{A}(\mathbb{B})$ acts on the subspace $S_{+}\left(S_{-}\right)$of positive (negative) chirality. See Appendix A for the irreducible representation of $\operatorname{Spin}(6)$ and $S U(4)$. Because classes $\mathbb{A}$ and $\mathbb{B}$ in (56) are now represented by $4 \times 4$ matrices on both sides, we can take a trace operation for the matrices which leads to the following relations:

$$
\begin{aligned}
& \mathbb{A}: R_{A B C D}^{(+)}=-F_{A B}^{(+) a} \operatorname{Tr}\left(T^{a} J_{+}^{C D}\right) \equiv F_{A B}^{(+) a} \eta_{C D}^{a} \\
& \mathbb{B}: R_{A B C D}^{(-)}=-F_{A B}^{(-) a} \operatorname{Tr}\left(\left(T^{a}\right)^{*} J_{-}^{C D}\right) \equiv F_{A B}^{(-) a} \bar{\eta}_{C D}^{a} .
\end{aligned}
$$

Here we have introduced a six-dimensional analogue of the 't Hooft symbols defined by

$$
\eta_{A B}^{( \pm) a}=-\operatorname{Tr}\left(T_{ \pm}^{a} J_{ \pm}^{A B}\right)
$$

where we used a bookkeeping notation, $\eta_{A B}^{(+) a} \equiv \eta_{A B}, \eta_{A B}^{(-) a} \equiv$ $\bar{\eta}_{A B}$, and $T_{+}^{a} \equiv T^{a}, T_{-}^{a} \equiv\left(T^{a}\right)^{*}$. They serve as a complete basis of the vector space 15 in (45) and (46). An explicit expression of the six-dimensional 't Hooft symbols and their algebra are presented in Appendix B.

Note that $F^{( \pm) a}=(1 / 2) F_{A B}^{( \pm) a} e^{( \pm) A} \wedge e^{( \pm) B}$ in (56) are the field strengths of $S U(4)$ gauge fields. Thus we introduce a pair of $S U(4)$ gauge fields $\left(A^{(+)}, A^{(-)}\right)$whose field strengths are given by

$$
F^{( \pm)}=d A^{( \pm)}+A^{( \pm)} \wedge A^{( \pm)}
$$

The SU(4) gauge field $A^{(+)}\left(A^{(-)}\right)$is nothing but the spin connection resident in the vector space $S_{+}\left(S_{-}\right)$of positive (negative) chirality; that is,

$$
\omega^{( \pm)}=\frac{1}{2} \omega_{A B}^{( \pm)} J_{ \pm}^{A B} \cong A^{( \pm)}=A^{( \pm) a} T_{ \pm}^{a}
$$

Using (B.7), the field strengths can be written as $F_{A B}^{( \pm) a}=$ $R_{A B C D}^{( \pm)} \eta_{C D}^{( \pm) a}=\eta_{C D}^{( \pm) a} R_{C D A B}^{( \pm)}$. One can apply again the same expansion to the index pair $[A B]$ of the Riemann tensor
$R_{C D A B}^{( \pm)}$. That is, one can expand the $S U(4)$ field strengths in terms of the chiral bases in (59):

$$
\begin{aligned}
& \mathbb{A}: F_{A B}^{(+) a}=f_{(++)}^{a b} \eta_{\mathrm{AB}}^{b}, \\
& \mathbb{B}: F_{A B}^{(-) a}=f_{(-)}^{a b} \bar{\eta}_{A B}^{b} .
\end{aligned}
$$

As was pointed out in (41), the Clifford algebra (40) is isomorphic to the exterior algebra $\Lambda^{*} M$ as vector spaces, so the 't Hooft symbol in (59) has a one-to-one correspondence with the basis of two forms in $\Omega_{ \pm}^{2}(M)=\Omega^{2}(M) \oplus * \Omega^{4}(M)$ depending on the chirality for a given orientation. Consequently, the six-dimensional Riemann curvature tensors can be expanded as follows:

$$
\begin{aligned}
& \mathbb{A}: R_{A B C D}^{(+)}=f_{(++)}^{a b} \eta_{A B}^{a} \eta_{C D}^{b}, \\
& \mathbb{B}: R_{A B C D}^{(-)}=f_{(--)}^{a b} \bar{\eta}_{A B}^{a} \bar{\eta}_{C D}^{b} .
\end{aligned}
$$

Note that the index pairs $[A B]$ and $[C D]$ in the curvature tensor $R_{A B C D}^{( \pm)}$have the same chirality structure because of the symmetry property $R_{A B C D}^{( \pm)}=R_{C D A B}^{( \pm)}$.

The Riemann curvature tensor in six dimensions has $225=15 \times 15$ components in total which is the number of the expansion coefficients $f_{( \pm \pm)}^{a b}$ in each class. Because the torsionfree condition has been assumed for the curvature tensors, the first Bianchi identity $R_{A[B C D]}^{( \pm)}=0$ should be imposed which leads to 120 constraints for each class. After all, the curvature tensor has $105=225-120$ independent components which must be equal to the number of remaining expansion coefficients in class $\mathbb{A}$ or $\mathbb{B}$ after solving the 120 constraints:

$$
\varepsilon^{A B C E F G} R_{D E F G}^{( \pm)}=0 .
$$

It is worthwhile to notice that the curvature tensor automatically satisfies the symmetry property $R_{A B C D}^{( \pm)}=R_{C D A B}^{( \pm)}$after dictating the first Bianchi identity (66). Therefore, one can split the 120 constraints in $(66)$ into the $105=(15 \times 14) / 2$ conditions imposing the symmetry $R_{A B C D}^{( \pm)}=R_{C D A B}^{( \pm)}$and the extra 15 conditions. These extra conditions can be manifest by considering the tensor product of $S U(4)$ [20]:

$$
15 \otimes 15=(1+15+20+84)_{S} \oplus(15+45+\overline{45})_{A S},
$$

where the first part with 120 components is symmetric and the second part with 105 components is antisymmetric. It is obvious from our construction that $f_{( \pm \pm)}^{a b} \in \mathbf{1 5} \otimes \mathbf{1 5}$. The 84 components in the symmetric part is the number of Weyl tensors in six dimensions and the $21=20+1$ components refer to Ricci tensors. The remaining 15 components in the symmetric part are removed by the first Bianchi identity (66) after expelling the antisymmetric components in (67).

One can easily solve the symmetry property $R_{A B C D}^{( \pm)}=$ $R_{C D A B}^{( \pm)}$with the coefficients satisfying

$$
\begin{aligned}
& f_{(++)}^{a b}=f_{(++)}^{b a}, \\
& f_{(--)}^{a b}=f_{(--)}^{b a},
\end{aligned}
$$


which results in 120 components for each chirality belonging to the symmetric part in (67). Now the remaining 15 conditions can be reduced to the equations

$$
\varepsilon^{A B C D E F} R_{C D E F}^{( \pm)}=0 .
$$

It is obvious that (69) gives rise to a nontrivial relation only for the coefficients satisfying (68). Finally, using (B.9) and (B.10), (69) can be reduced to the 15 constraints

$$
d^{a b c} f_{(++)}^{b c}=d^{a b c} f_{(--)}^{b c}=0
$$

for each sector. In the end, $f_{( \pm \pm)}^{a b}$ have 105 independent components for each chirality which precisely match with the independent components of Riemann curvature tensors in class $\mathbb{A}$ or $\mathbb{B}$. (It may be worthwhile to recall the fourdimensional situation $[12,13]$. In four dimensions, the first Bianchi identity gives rise to 16 constraints. Thus Riemann curvature tensors have $20=36-16$ independent components. And the 16 constraints split into 15 ones for $R_{A B C D}=$ $R_{C D A B}$ and one more constraint which reads as $\delta^{a b} f_{(++)}^{a b}=$ $\delta^{\dot{a} \dot{b}} f_{(--)}^{\dot{a} \dot{b}}$. The last constraint is responsible for the equality of the Ricci scalar $s$ in the chiral and antichiral sectors in (8). The constraints in (70) correspond to the six-dimensional analogue of the last one.)

Let us introduce the following (projection) operator acting on $6 \times 6$ antisymmetric matrices defined by

$$
\begin{aligned}
P_{ \pm}^{A B C D} & \equiv \frac{1}{4}\left(\delta_{A C} \delta_{B D}-\delta_{A D} \delta_{B C}\right) \pm \frac{1}{8} \varepsilon^{A B C D E F} I_{E F} \\
& =P_{ \pm}^{C D A B},
\end{aligned}
$$

where $I \equiv \mathbf{I}_{3} \otimes i \sigma^{2}$. Because any $6 \times 6$ antisymmetric matrix of rank 4 spans a four-dimensional subspace $\mathbb{R}^{4} \subset \mathbb{R}^{6}$, the operator (71) in this case can be written in the fourdimensional subspace as

$$
P_{ \pm}^{A B C D} \equiv \frac{1}{4}\left(\delta_{A C} \delta_{B D}-\delta_{A D} \delta_{B C}\right) \pm \frac{1}{4} \varepsilon^{A B C D},
$$

$$
(A, B, C, D) \in \mathbb{R}^{4},
$$

so it reduces to the projection operator for such rank 4 matrices; that is,

$$
\begin{aligned}
& P_{ \pm}^{A B E F} P_{ \pm}^{E F C D}=P_{ \pm}^{A B C D}, \\
& P_{ \pm}^{A B E F} P_{\mp}^{E F C D}=0 .
\end{aligned}
$$

Note that $I_{A B}$ is a $6 \times 6$ antisymmetric matrix of rank 6 . In this case, the operator (71) does not act as a projection operator but acts as

$$
P_{ \pm}^{A B C D} I_{C D}=\left(\frac{1}{2} \pm 1\right) I_{A B}
$$

In general, one can deduce by a straightforward calculation the following properties:

$$
\begin{aligned}
& P_{ \pm}^{A B E F} P_{ \pm}^{E F C D}=P_{ \pm}^{A B C D}+\frac{1}{8} I_{A B} I_{C D}, \\
& P_{ \pm}^{A B E F} P_{\mp}^{E F C D}=-\frac{1}{8} I_{A B} I_{C D} .
\end{aligned}
$$

After a little algebra, one can classify the 't Hooft symbols in (59) into the eigenspaces of the operator (71):

$$
\begin{aligned}
l_{A B}^{(+) \widehat{a}} \equiv\left\{\eta_{A B}^{13}=\frac{i}{2} \lambda_{1} \otimes \sigma^{2}, \eta_{A B}^{14}=\frac{i}{2} \lambda_{2}\right. \\
\otimes \mathbf{I}_{2}, \frac{1}{\sqrt{3}}\left(\eta_{A B}^{8}-\sqrt{2} \eta_{A B}^{15}\right)=-\frac{i}{2} \lambda_{3} \otimes \sigma^{2}, \eta_{A B}^{6} \\
=\frac{i}{2} \lambda_{4} \otimes \sigma^{2}, \eta_{A B}^{7}=-\frac{i}{2} \lambda_{5} \otimes \mathbf{I}_{2}, \eta_{A B}^{11}=\frac{i}{2} \lambda_{6} \\
\otimes \sigma^{2}, \eta_{A B}^{12}=-\frac{i}{2} \lambda_{7} \\
\otimes \mathbf{I}_{2}, \frac{2}{\sqrt{3}}\left(-\frac{1}{2} \eta_{A B}^{3}+\frac{1}{\sqrt{3}} \eta_{A B}^{8}+\frac{1}{\sqrt{6}} \eta_{A B}^{15}\right)=-\frac{i}{2} \lambda_{8} \\
\left.\otimes \sigma^{2}\right\}, \\
m_{A B}^{(+) \dot{a}} \equiv\left\{\eta_{A B}^{1}=\frac{i}{2} \lambda_{2} \otimes \sigma^{1}, \eta_{A B}^{2}=-\frac{i}{2} \lambda_{2} \otimes \sigma^{3}, \eta_{A B}^{9}\right. \\
\quad=-\frac{i}{2} \lambda_{5} \otimes \sigma^{1}, \eta_{A B}^{10}=\frac{i}{2} \lambda_{5} \otimes \sigma^{3}, \eta_{A B}^{4}=\frac{i}{2} \lambda_{7} \\
\left.\otimes \sigma^{1}, \eta_{A B}^{5}=-\frac{i}{2} \lambda_{7} \otimes \sigma^{3}\right\}, \\
n_{A B}^{(+) 0} \equiv\left\{\eta_{A B}^{3}+\frac{1}{\sqrt{3}} \eta_{A B}^{8}+\frac{1}{\sqrt{6}} \eta_{A B}^{15}=\frac{1}{2} I_{A B}=\frac{1}{2} \mathbf{I}_{3}\right. \\
\otimes
\end{aligned}
$$

where $\widehat{a}, \widehat{b}=1, \ldots, 8$ and $\dot{a}, \dot{b}=1, \ldots, 6$ are $s u(4)$ indices in the entries of $l_{A B}^{(+)}$and $m_{A B}^{(+)}$, respectively. They obey the following relations:

$$
\begin{aligned}
P_{-}^{A B C D} l_{C D}^{(+) \hat{a}} & =l_{A B}^{(+) \hat{a}}, \\
P_{+}^{A B C D} l_{C D}^{(+) \widehat{a}} & =0, \\
P_{-}^{A B C D} m_{C D}^{(+) \dot{a}} & =0, \\
P_{+}^{A B C D} m_{C D}^{(+) \dot{a}} & =m_{A B}^{(+) \dot{a}}, \\
P_{-}^{A B C D} n_{C D}^{(+) 0} & =-\frac{1}{2} n_{A B}^{(+) 0}, \\
P_{+}^{A B C D} n_{C D}^{(+) 0} & =\frac{3}{2} n_{A B}^{(+) 0} .
\end{aligned}
$$

Thus the (projection) operators (71) decompose the vector space 15 into their eigenspaces as $\mathbf{1 5}=\mathbf{8} \oplus \mathbf{6} \oplus \mathbf{1}$.

Similarly, one can also classify the 't Hooft symbols in (B.2) into the eigenspaces of the operator (71):

$$
\begin{aligned}
1_{A B}^{(-) \widehat{a}} & \equiv\left\{-\bar{\eta}_{A B}^{13}=\frac{i}{2} \lambda_{1} \otimes \sigma^{2}, \bar{\eta}_{A B}^{14}=\frac{i}{2} \lambda_{2}\right. \\
& \otimes \mathbf{I}_{2}, \frac{1}{\sqrt{3}}\left(-\bar{\eta}_{A B}^{8}+\sqrt{2} \bar{\eta}_{A B}^{15}\right)=-\frac{i}{2} \lambda_{3} \otimes \sigma^{2}, \quad-\bar{\eta}_{A B}^{9}
\end{aligned}
$$




$$
\begin{gathered}
=\frac{i}{2} \lambda_{4} \otimes \sigma^{2}, \quad-\bar{\eta}_{A B}^{10}=-\frac{i}{2} \lambda_{5} \otimes \mathbf{I}_{2}, \bar{\eta}_{A B}^{4}=\frac{i}{2} \lambda_{6} \\
\otimes \sigma^{2}, \bar{\eta}_{A B}^{5}=-\frac{i}{2} \lambda_{7} \\
\otimes \mathbf{I}_{2}, \frac{2}{\sqrt{3}}\left(\frac{1}{2} \bar{\eta}_{A B}^{3}+\frac{1}{\sqrt{3}} \bar{\eta}_{A B}^{8}+\frac{1}{\sqrt{6}} \bar{\eta}_{A B}^{15}\right)=-\frac{i}{2} \lambda_{8} \\
\left.\otimes \sigma^{2}\right\}, \\
m_{A B}^{(-) \dot{a}} \equiv\left\{-\bar{\eta}_{A B}^{1}=\frac{i}{2} \lambda_{2} \otimes \sigma^{1}, \bar{\eta}_{A B}^{2}=-\frac{i}{2} \lambda_{2} \otimes \sigma^{3},\right. \\
-\bar{\eta}_{A B}^{6}=-\frac{i}{2} \lambda_{5} \otimes \sigma^{1},-\bar{\eta}_{A B}^{7}=\frac{i}{2} \lambda_{5} \otimes \sigma^{3}, \bar{\eta}_{A B}^{11} \\
\left.=\frac{i}{2} \lambda_{7} \otimes \sigma^{1}, \bar{\eta}_{A B}^{12}=-\frac{i}{2} \lambda_{7} \otimes \sigma^{3}\right\}, \\
n_{A B}^{(-) 0} \equiv\left\{-\bar{\eta}_{A B}^{3}+\frac{1}{\sqrt{3}} \bar{\eta}_{A B}^{8}+\frac{1}{\sqrt{6}} \bar{\eta}_{A B}^{15}=\frac{1}{2} I_{A B}=\frac{1}{2} \mathbf{I}_{3}\right. \\
\left.\otimes i \sigma^{2}\right\} .
\end{gathered}
$$

The same properties such as (79) also hold for the above 't Hooft symbols.

The geometrical meaning of the (projection) operators in (71) can be understood as follows. Consider an arbitrary two-form vector space (we will indicate the superscript $(+)$ or $(-)$ only when we refer to a quantity belonging to a definite chirality class; we will often omit the superscript whenever it is not necessary to specify the chirality class)

$$
F=\frac{1}{2} F_{M N} d x^{M} \wedge d x^{N}=\frac{1}{2} F_{A B} e^{A} \wedge e^{B} \in \Omega^{2}(M)
$$

and introduce the 15-dimensional complete basis of two forms in $\Omega_{ \pm}^{2}(M)$ for each chirality of spin(6) Lorentz algebra

$$
\begin{aligned}
J_{+}^{a} & \equiv \frac{1}{2} \eta_{A B}^{a} e^{(+) A} \wedge e^{(+) B} \in \Omega_{+}^{2}(M), \\
J_{-}^{a} & \equiv \frac{1}{2} \bar{\eta}_{A B}^{a} e^{(-) A} \wedge e^{(-) A} \in \Omega_{-}^{2}(M) .
\end{aligned}
$$

It is easy to derive the following identity using (B.9) and (B.10):

$$
J_{ \pm}^{a} \wedge J_{ \pm}^{b} \wedge J_{ \pm}^{c}=\frac{1}{2} d^{a b c} \operatorname{vol}\left(g^{( \pm)}\right)
$$

where $\operatorname{vol}\left(g^{( \pm)}\right)=\sqrt{g^{( \pm)}} d^{6} x$. The Hodge-dual operator $*$ : $\Omega^{k}(M) \rightarrow \Omega^{6-k}(M)$ is an isomorphism of vector spaces which depends upon a metric $g^{( \pm)}$and the orientation of $M$. The nowhere vanishing volume form in (83) guarantees that there exists a set of nondegenerate 2 -form vector spaces on $M$

$$
\begin{aligned}
\Omega_{ \pm} & =\frac{1}{2} I_{A B} e^{( \pm) A} \wedge e^{( \pm) B} \\
& =e^{( \pm) 1} \wedge e^{( \pm) 2}+e^{( \pm) 3} \wedge e^{( \pm) 4}+e^{( \pm) 5} \wedge e^{( \pm) 6} .
\end{aligned}
$$

This two-form vector space can be wedged with the Hodge star to construct a diagonalizable operator on $\Lambda^{2}(M)=$ $\Omega^{2}(M) \oplus * \Omega^{4}(M)$ as follows:

$$
{ }^{*} \Omega_{ \pm} \equiv *\left(\bullet \wedge \Omega_{ \pm}\right): \Omega^{2}(M) \stackrel{\bullet \wedge \Omega_{ \pm}}{\longrightarrow} \Omega^{4}(M) \stackrel{*}{\rightarrow} \Omega^{2}(M)
$$

by $*_{\Omega_{+}}(\alpha)=*\left(\alpha \wedge \Omega_{ \pm}\right)$for $\alpha \in \Omega^{2}(M)$. After a little inspection, the $15 \times 15$ matrix representing $*_{\Omega_{+}}$is found to have the eigenvalues 2,1 and -1 with the eigenspaces of dimensions 1,6 , and 8 , respectively. On any six-dimensional orientable spin manifold $M$, the space of 2 -form $\Omega_{+}^{2}(M)$ in the positive chirality space can thus be decomposed into three subspaces:

$$
\Omega_{+}^{2}(M)=\Lambda_{1}^{2} \oplus \Lambda_{6}^{2} \oplus \Lambda_{8}^{2},
$$

which coincides with the decomposition in (79). The spaces $\Lambda_{1}^{2}$ and $\Lambda_{6}^{2}$ are locally spanned by

$$
\begin{aligned}
& \Lambda_{1}^{2}=\Omega_{+}, \\
& \Lambda_{6}^{2}=\left\{J_{+}^{1}, J_{+}^{2}, J_{+}^{4}, J_{+}^{5}, J_{+}^{9}, J_{+}^{10}\right\}
\end{aligned}
$$

and $\Lambda_{8}^{2}$ by

$$
\Lambda_{8}^{2}=\left\{J_{+}^{6}, J_{+}^{7}, J_{+}^{11}, J_{+}^{12}, J_{+}^{13}, J_{+}^{14}, K_{+}, L_{+}\right\}
$$

with $K_{+} \equiv(1 / \sqrt{3})\left(J_{+}^{8}-\sqrt{2} J_{+}^{15}\right)$ and $L_{+} \equiv(2 / \sqrt{3})\left(-(1 / 2) J_{+}^{3}+\right.$ $\left.(1 / \sqrt{3}) J_{+}^{8}+(1 / \sqrt{6}) J_{+}^{15}\right)$. A similar decomposition can be done with the negative chirality basis $J_{-}^{a}$.

Note that the entries of $\Lambda_{1}^{2}, \Lambda_{6}^{2}$, and $\Lambda_{8}^{2}$ coincide with those of $n_{A B}^{( \pm) 0}, m_{A B}^{( \pm) \dot{a}}$, and $l_{A B}^{( \pm) \widehat{a}}$, respectively. One can quickly see that this coincidence is not an accident. Consider the action of the projection operator (71) on the two-form (81), which is given by

$$
P_{ \pm}^{A B C D} F_{C D}=\frac{1}{2}\left(F_{A B} \pm \frac{1}{4} \varepsilon^{A B C D E F} F_{C D} I_{E F}\right)
$$

or in terms of form notation

$$
2 P_{ \pm} F=F \pm *\left(F \wedge \Omega_{ \pm}\right)=F \pm *_{\Omega_{ \pm}} F .
$$

It is easy to see that $F \in \Lambda_{8}^{2}$ if $P_{+} F=0$, so it satisfies the $\Omega$-anti-self-duality equation

$$
*\left(F \wedge \Omega_{ \pm}\right)=-F
$$

whereas $F \in \Lambda_{6}^{2}$ satisfies the $\Omega$-self-duality equation $P_{-} F=0$; that is,

$$
*\left(F \wedge \Omega_{ \pm}\right)=F
$$

It is not difficult to show [1] that the set $\left\{l_{A B}^{( \pm) \widehat{a}}, n_{A B}^{( \pm) 0}\right\}$ can be identified with $u(3)$ generators which are embedded in $s o(6) \cong s u(4)$. In general, an element of $U(3)$ group can be represented as

$$
U=\exp \left(i \sum_{a=0}^{8} \theta^{a} \lambda_{a}\right) \equiv e^{\Theta},
$$


where $\lambda_{0}=\mathbf{I}_{3}$ is a $3 \times 3$ unit matrix, $\lambda_{\widehat{a}}(\widehat{a}=1, \ldots, 8)$ are the $s u(3)$ Gell-Mann matrices, and $\theta^{a}$ s are real parameters for $U$ to be unitary. The $3 \times 3$ anti-Hermitian matrix $\Theta$ consists of matrix elements which are complex numbers $\Theta_{i \bar{j}}=$ $-\left(\Theta_{\overline{j i}}\right)^{*}(i, \bar{j}=1,2,3)$ and it can easily be embedded into a $6 \times 6$ real matrix in so(6) Lie algebra by replacing $\Theta_{i \bar{j}}=$ $\operatorname{Re} \Theta_{i \bar{j}}+i \operatorname{Im} \Theta_{i \bar{j}}$ by the $2 \times 2$ real matrix $\widetilde{\Theta}_{A B}=\mathrm{I}_{2} \cdot \operatorname{Re} \Theta_{i \bar{j}}+i \sigma^{2}$. $\operatorname{Im} \Theta_{i \bar{j}}$. A straightforward calculation (see (B.17)) shows that the resulting $6 \times 6$ antisymmetric real matrix $\widetilde{\Theta}_{A B}$ can be written as

$$
\widetilde{\Theta}_{A B}=2\left(\theta^{0} n_{A B}^{( \pm) 0}+\theta^{\widehat{a}} l_{A B}^{( \pm) \widehat{a}}\right)=P_{-}^{A B C D} \widetilde{\Theta}_{C D}+3 \theta^{0} n_{A B}^{( \pm) 0} .
$$

Note that $U(3)$ is the holonomy group of Kähler manifolds. That is, the projection operators in (71) can serve to project a Riemannian manifold whose holonomy group is $S O(6)$ into a Kähler manifold with $U(3)$ holonomy. Let us show that it is indeed the case. Suppose that $M$ is a complex manifold. Let us introduce local complex coordinates $z^{\alpha}=$ $\left\{x^{1}+i x^{2}, x^{3}+i x^{4}, x^{5}+i x^{6}\right\}, \alpha=1,2,3$ and their complex conjugates $\bar{z}^{\bar{\alpha}}, \bar{\alpha}=1,2,3$, in which a complex structure $J$ takes the form $J_{\beta}^{\alpha}=i \delta_{\beta}^{\alpha}, J^{\bar{\alpha}} \bar{\beta}=-i \delta^{\bar{\alpha}} \bar{\beta}[1]$. Note that, relative to the real basis $x^{M}, M=1, \ldots, 6$, the complex structure is given by $J=I=\mathbf{I}_{3} \otimes i \sigma^{2}$ which was already introduced in (71). We further impose the Hermitian condition on the complex manifold $M$ defined by $g(X, Y)=g(J X, J Y)$ for any $X, Y \in \Gamma(T M)$. This means that the Riemannian metric $g$ on the complex manifold $M$ is a Hermitian metric; that is, $g_{\alpha \beta}=$ $g_{\bar{\alpha} \bar{\beta}}=0, g_{\alpha \bar{\beta}}=g_{\bar{\beta} \alpha}$. The Hermitian condition can be solved by taking the vielbeins as

$$
\begin{aligned}
& e_{\bar{\alpha}}^{i}=e_{\alpha}^{\bar{i}}=0, \\
& E_{i}^{\bar{\alpha}}=E_{\bar{i}}^{\alpha}=0,
\end{aligned}
$$

where a tangent space index $A=1, \ldots, 6$ has been split into a holomorphic index $i=1,2,3$ and an antiholomorphic index $\bar{i}=1,2,3$. This in turn means that $J^{i}{ }_{j}=i \delta^{i}{ }_{j}, J^{\bar{i}}{ }_{j}=-i \delta^{\bar{i}}{ }_{\bar{j}}$. Then one can see that the two-form $\Omega_{+}$in (84) is a Kähler form; that is, $\Omega_{ \pm}(X, Y)=g^{( \pm)}(J X, Y)$, and it is given by

$$
\begin{aligned}
\Omega_{ \pm} & =i e^{( \pm) i} \wedge e^{( \pm) \bar{i}}=i e_{\alpha}^{( \pm) i} e_{\bar{\beta}}^{( \pm) \bar{i}} d z^{\alpha} \wedge d \bar{z}^{\bar{\beta}} \\
& =i g_{\alpha \bar{\beta}}^{( \pm)} d z^{\alpha} \wedge d \bar{z}^{\bar{\beta}}
\end{aligned}
$$

where $e^{( \pm) i}=e_{\alpha}^{( \pm) i} d z^{\alpha}$ is holomorphic one form and $e^{( \pm) \bar{i}}=$ $e_{\bar{\alpha}}^{( \pm) \bar{i}} d \bar{z}^{\bar{\alpha}}$ is antiholomorphic one form. It is also easy to see that the condition for a Hermitian manifold $\left(M, g^{( \pm)}\right)$to be Kähler, that is, $d \Omega_{ \pm}=0$, is equivalent to the one where the spin connection $\omega_{A B}^{( \pm)}$is $U(3)$-valued; that is,

$$
\omega_{i j}^{( \pm)}=\omega_{\overline{i j}}^{( \pm)}=0
$$

Therefore, the spin connection after the Kähler condition (97) can be written as the form (94).
All the above results can be clearly understood by the properties of Spin(6) and SU(4) groups. Introducing complex coordinates on $\mathbb{R}^{6}$ means that one has to consider the Lorentz subgroup $U(3) \subset S U(4)$ acting on $\mathbb{C}^{3} \subset \mathbb{C}^{4}$ and so one decomposes the $\mathbf{4}$ and $\overline{\mathbf{4}}$ of $S U(4)$ as $\mathbf{4}=\mathbf{1}_{1} \oplus \mathbf{3}_{-1 / 3}$ and $\overline{\mathbf{4}}=\overline{\mathbf{1}}_{-1} \oplus \overline{\mathbf{3}}_{1 / 3}$ under $U(3)=U(1) \times S U(3)$ where the subscripts denote $U(1)$ charges. Using the branching rule of $S U(4)>$ $U(1) \times S U(3)$ [20], one can get the following decompositions after removing $S U(4)$ singlets:

$$
\begin{aligned}
\mathbf{4} \otimes \overline{\mathbf{4}}-\mathbf{1} & =(\mathbf{3} \otimes \overline{\mathbf{3}})_{0} \oplus\left(\mathbf{3}_{-4 / 3} \oplus \overline{\mathbf{3}}_{4 / 3}\right) \\
& =(\mathbf{8} \oplus \mathbf{1})_{0} \oplus\left(\mathbf{3}_{-4 / 3} \oplus \overline{\mathbf{3}}_{4 / 3}\right), \\
\overline{\mathbf{4}} \otimes \mathbf{4}-\mathbf{1} & =(\overline{\mathbf{3}} \otimes \mathbf{3})_{0} \oplus\left(\mathbf{3}_{-4 / 3} \oplus \overline{\mathbf{3}}_{4 / 3}\right) \\
& =(\mathbf{8} \oplus \mathbf{1})_{0} \oplus\left(\mathbf{3}_{-4 / 3} \oplus \overline{\mathbf{3}}_{4 / 3}\right) .
\end{aligned}
$$

The spin connection $\omega_{A B} \in \mathbf{1 5}$ can be decomposed according to the above branching rule as

$$
\begin{aligned}
\omega_{i j} & \in \mathbf{3}_{-4 / 3}, \\
\omega_{\overline{i j}} & \in \overline{\mathbf{3}}_{4 / 3}, \\
\omega_{\bar{i} j}-\frac{1}{3} \delta_{j}^{i} \omega_{\bar{k} k} & \in \mathbf{8}_{0}, \\
\omega_{\bar{i} i} & \in \mathbf{1}_{0} .
\end{aligned}
$$

Hence the Kähler condition (97) means that spin connections in $\mathbf{3}_{-4 / 3}$ and $\overline{\mathbf{3}}_{4 / 3}$ decouple from the theory and only the components in $\mathbf{8}_{0}$ and $\mathbf{1}_{0}$ survive. It is now obvious why we could have such decompositions in (76)-(80) in which $l_{A B}^{( \pm) \widehat{a}} \in$ $\mathbf{8}_{0}, m_{A B}^{( \pm) \dot{a}} \in\left(\mathbf{3}_{-4 / 3} \oplus \overline{\mathbf{3}}_{4 / 3}\right)$, and $n_{A B}^{( \pm) 0} \in \mathbf{1}_{0}$.

One can rephrase the Kähler condition (97) using the gauge theory formalism. From the identification $\omega^{( \pm)} \equiv$ $\Gamma_{ \pm} \oplus=A^{( \pm) a} T_{ \pm}^{a}$ in (61), we get the relation

$$
\omega_{A B}^{( \pm)}=A^{( \pm) a} \eta_{A B}^{( \pm) a}, \quad A^{( \pm) a}=-2 \operatorname{Tr}\left(\omega^{( \pm)} T_{ \pm}^{a}\right) .
$$

We will focus on type $\mathbb{A}$ case as the same analysis can be applied to type $\mathbb{B}$ case. If $\left(M, g^{(+)}\right)$is a Kähler manifold, (97) means that

$$
A^{(+) 1}=A^{(+) 2}=A^{(+) 4}=A^{(+) 5}=A^{(+) 9}=A^{(+) 10}=0
$$

because $\eta_{i j}^{a} \neq 0$ only for $a=1,2,4,5,9,10$; otherwise, $\eta_{i j}^{a}=0$. See (B.17). This result is consistent with the branching rule (99); that is, $m_{A B}^{(+) \dot{a}} \in\left(\mathbf{3}_{-4 / 3} \oplus \overline{\mathbf{3}}_{4 / 3}\right)$. In other words, $A^{(+) \dot{a}}=$ 0 and so the gauge fields take values in $u(3)$ Lie algebra according to the result (94). Then the SU(4) structure constants $f^{a b c}$ in Table 1 guarantee that the corresponding field strengths also vanish; that is,

$$
F^{(+) a}=\frac{1}{2} f_{(++)}^{a b} \eta_{A B}^{b} e^{(+) A} \wedge e^{(+) B}=0
$$


TABLE 1: The nonvanishing structure constants $f^{a b c}$.

\begin{tabular}{|c|c|c|c|}
\hline$a$ & $b$ & $c$ & $f^{a b c}$ \\
\hline 1 & 2 & 3 & 1 \\
\hline 1 & 4 & 7 & $1 / 2$ \\
\hline 1 & 5 & 6 & $-1 / 2$ \\
\hline 1 & 9 & 12 & $1 / 2$ \\
\hline 1 & 10 & 11 & $-1 / 2$ \\
\hline 2 & 4 & 6 & $1 / 2$ \\
\hline 2 & 5 & 7 & $1 / 2$ \\
\hline 2 & 9 & 11 & $1 / 2$ \\
\hline 2 & 10 & 12 & $1 / 2$ \\
\hline 3 & 4 & 5 & $1 / 2$ \\
\hline 3 & 6 & 7 & $-1 / 2$ \\
\hline 3 & 9 & 10 & $1 / 2$ \\
\hline 3 & 11 & 12 & $-1 / 2$ \\
\hline 4 & 5 & 8 & $\sqrt{3} / 2$ \\
\hline 4 & 9 & 14 & $1 / 2$ \\
\hline 4 & 10 & 13 & $-1 / 2$ \\
\hline 5 & 9 & 13 & $1 / 2$ \\
\hline 5 & 10 & 14 & $1 / 2$ \\
\hline 6 & 7 & 8 & $\sqrt{3} / 2$ \\
\hline 6 & 11 & 14 & $1 / 2$ \\
\hline 6 & 12 & 13 & $-1 / 2$ \\
\hline 7 & 11 & 13 & $1 / 2$ \\
\hline 7 & 12 & 14 & $1 / 2$ \\
\hline 8 & 9 & 10 & $1 / 2 \sqrt{3}$ \\
\hline 8 & 11 & 12 & $1 / 2 \sqrt{3}$ \\
\hline 8 & 13 & 14 & $-1 / \sqrt{3}$ \\
\hline 9 & 10 & 15 & $\sqrt{2 / 3}$ \\
\hline 11 & 12 & 15 & $\sqrt{2 / 3}$ \\
\hline 13 & 14 & 15 & $\sqrt{2 / 3}$ \\
\hline
\end{tabular}

for $a=1,2,4,5,9,10$. Thus we get $f_{(++)}^{\dot{a} b}=0$ for $\forall b=$ $1, \ldots, 15$. This immediately leads to the conclusion that

$$
\begin{aligned}
F^{(+) a} & =d A^{(+) a}-\frac{1}{2} f^{a b c} A^{(+) b} \wedge A^{(+) c}=f_{(++)}^{a b} J_{+}^{b} \\
& \in \Lambda_{8}^{2} \oplus \Lambda_{1}^{2},
\end{aligned}
$$

where $F^{(+) a}, a=0,1, \ldots, 8$, are the field strengths of $U(3)$ gauge fields. As will be shown below, $F^{(+) 0} \in \Lambda_{1}^{2}$ is the field strength of the $U(1)$ part of $U(3)$ spin connections and $F^{(+) \widehat{a}} \in$ $\Lambda_{8}^{2}, \widehat{a}=1, \ldots, 8$, belong to the $S U(3)$ part. In particular, as $F^{(+) \widehat{a}} \in \Lambda_{8}^{2}$, they satisfy the $\Omega$-anti-self-duality equation (91) known as the HYM equation $[17,18]$

$$
F^{(+) \hat{a}}=-*\left(F^{(+) \hat{a}} \wedge \Omega_{+}\right), \quad \widehat{a}=1, \ldots, 8 .
$$

It is well-known [1] that the Ricci-tensor of a Kähler manifold is the field strength of the $U(1)$ part of the $U(3)$ spin connection. Therefore, the Ricci-flat condition can be stated as $F^{(+) 0}=0$. One can explicitly check it as follows. Recall that $F_{A B}^{(+) a}=f_{(++)}^{a b} \eta_{A B}^{b}$. Using the result (102), one can see that the nonzero components of $f_{(++)}^{a b}$ run only over $(a, b) \in\{3,6,7,8,11,12,13,14,15\}$. Thereby the constraint (70) becomes nontrivial only for those values. As a result, the number of independent components of $f_{(++)}^{a b}$ is given by $(9 \times 10) / 2-9=36$. The Ricci-flat condition $R_{A B}^{(+)} \equiv R_{A C B C}^{(+)}=$ $f_{(++)}^{a b} \eta_{A C}^{a} \eta_{B C}^{b}=0$ further constrains the coefficients. A close inspection shows that out of 21 equations, $R_{A B}^{(+)}=0$, only 9 equations are independent and, after utilizing the constraint (70), the equations for the Ricci-flatness can be succinctly arranged as

$$
f_{(++)}^{3 a}+\frac{1}{\sqrt{3}} f_{(++)}^{8 a}+\frac{1}{\sqrt{6}} f_{(++)}^{15 a}=0 .
$$

The above condition means that

$$
\begin{aligned}
F_{A B}^{(+) 0} & =\left(f_{(++)}^{3 a}+\frac{1}{\sqrt{3}} f_{(++)}^{8 a}+\frac{1}{\sqrt{6}} f_{(++)}^{15 a}\right) \eta_{A B}^{a} \\
& =F_{A B}^{(+) 3}+\frac{1}{\sqrt{3}} F_{A B}^{(+) 8}+\frac{1}{\sqrt{6}} F_{A B}^{(+) 15}=0 .
\end{aligned}
$$

If one introduces a gauge field defined by

$$
A^{(+) 0} \equiv \omega_{A B}^{(+)} n_{A B}^{(+) 0}=A^{(+) 3}+\frac{1}{\sqrt{3}} A^{(+) 8}+\frac{1}{\sqrt{6}} A^{(+) 15},
$$

one can show that the field strength in (106) is given by

$$
F^{(+) 0}=d A^{(+) 0}
$$

after using the fact that the $U(3)$ structure constants $f^{a b c}$ satisfy the following relation:

$$
f^{3 a b}+\frac{1}{\sqrt{3}} f^{8 a b}+\frac{1}{\sqrt{6}} f^{15 a b}=0 .
$$

The relation (109) is easy to understand because the $U(1)$ part among the $U(3)$ structure constants has to vanish. This establishes the result that the Ricci-flatness is equal to the vanishing of the $U(1)$ field strength. That is, $F^{(+) 0}=d A^{(+) 0} \epsilon$ $\Lambda_{1}^{2}$ has a trivial first Chern class.

The same result can be obtained for type $\mathbb{B}$ case. The Kähler condition (97) can similarly be solved by

$$
A^{(-) 1}=A^{(-) 2}=A^{(-) 6}=A^{(-) 7}=A^{(-) 11}=A^{(-) 12}=0 .
$$

Note that the entries of $U(3)$ generators for type $\mathbb{B}$ case are different from those for type $\mathbb{A}$ case. The Ricci-flat condition $R_{A B}^{(-)} \equiv R_{A C B C}^{(-)}=f_{(-)}^{a b} \bar{\eta}_{A C}^{a} \bar{\eta}_{B C}^{b}=0$ leads to the equation

$$
-f_{(--)}^{3 a}+\frac{1}{\sqrt{3}} f_{(--)}^{8 a}+\frac{1}{\sqrt{6}} f_{(--)}^{15 a}=0 .
$$

It is equivalent to the vanishing of $U(1)$ field strength; that is, $F^{(-) 0}=d A^{(-) 0}=0$, where the $U(1)$ gauge field is defined by

$$
A^{(-) 0} \equiv \omega_{A B}^{(-)} n_{A B}^{(-) 0}=-A^{(-) 3}+\frac{1}{\sqrt{3}} A^{(-) 8}+\frac{1}{\sqrt{6}} A^{(-) 15} .
$$

This fact can be derived by using the fact that the $U(3)$ structure constants $f^{a b c}$ for type $\mathbb{B}$ case satisfy the following relation:

$$
-f^{3 a b}+\frac{1}{\sqrt{3}} f^{8 a b}+\frac{1}{\sqrt{6}} f^{15 a b}=0,
$$


where $a, b$ now run over $3,4,5,8,9,10,13,14,15$. Hence one can see that CY manifolds for type $\mathbb{B}$ case also obey the HYM equations

$$
F^{(-) \widehat{a}}=-*\left(F^{(-) \widehat{a}} \wedge \Omega_{-}\right), \quad \widehat{a}=1, \ldots, 8 .
$$

Note that the HYM equations for a vector bundle $E$ over a CY manifold $M$ define a set of differential equations satisfied by the gauge fields of the vector bundle $E \rightarrow M$. In general, the connection of a vector bundle $E \rightarrow M$ over a CY manifold $M$ is not related to the spin connection of the CY manifold $M$ unless $E=T M$. For example, the $G$ bundle over a CY manifold $M$ is such a case. However, in our case, we have adopted the so-called standard embedding $E=T M$, where $T M$ is the tangent bundle of a CY manifold $M$. Then the gauge fields of $E=T M$ are the spin connections on the tangent bundle TM of the CY manifold $M$. Since the tangent bundle $T M$ is defined by the CY manifold $M$ itself, the HYM instanton in this case is inherited from the CY manifold. Therefore, the HYM instanton for the tangent bundle TM cannot be identified with an ordinary Yang-Mills instanton on a fixed background manifold since the YangMills connection of TM is directly determined by the CY manifold $M$.

In summary, the Kähler condition (97) projects the 't Hooft symbols to $U(3)$-valued ones in $\mathbf{1}_{0} \oplus \mathbf{8}_{0}$ and results in the reduction of the gauge group from $S U(4)$ to $U(3)$. The Ricciflatness is equivalent to the condition $F^{( \pm) 0}=d A^{( \pm) 0}=0 \in \mathbf{1}_{0}$, so the gauge group is further reduced to $S U(3)$. Remaining spin connections are $S U(3)$ gauge fields that belong to $\mathbf{8}_{0}$ and satisfy the HYM (104) or (114). As a Kähler manifold with the trivial first Chern class is a CY manifold, we see that the CY condition is equivalent to the HYM equations whose solution is known as HYM instantons [1]. Consequently, we find that a six-dimensional CY manifold automatically satisfies the HYM equations in SU(3) Yang-Mills gauge theory, but the converse is not generally true.

\section{Mirror Symmetry of Calabi-Yau Manifolds}

In this section we want to explore the geometrical properties of six-dimensional Riemannian manifolds in the irreducible representations $\mathbb{A}$ and $\mathbb{B}$. In Section 2, we have introduced dual vielbeins $\widetilde{e}^{A}=(* h)^{A}$ and dual spin connections $\widetilde{\omega}_{A B}=(* \theta)_{A B}$ in addition to usual ones $\left(e^{A}, \omega_{A B}\right)$. The dual geometric structure described by $\left(\widetilde{e}^{A}, \widetilde{\omega}_{A B}\right) \cong\left(h^{A}, \theta_{A B}\right)$ is basically originated from the Hodge duality of the exterior algebra $\Lambda^{*} M$ on an orientable manifold $M$. According to the chiral structure of irreducible representations in (45)-(48), we have associated two geometric structures $\left(e^{(+) A}, \omega_{A B}^{(+)}\right)$and $\left(e^{(-) A}, \omega_{A B}^{(-)}\right)$on a spin manifold $M$ where

$$
\begin{aligned}
e^{( \pm) A} & =\frac{1}{2}(e \pm \widetilde{e})^{A}=\frac{1}{2}(e \pm * h)^{A}, \\
\omega_{A B}^{( \pm)} & =\frac{1}{2}(\omega \pm \widetilde{\omega})_{A B}=\frac{1}{2}(\omega \pm * \theta)_{A B} .
\end{aligned}
$$

This means that there are two independent ways to characterize a six-dimensional spin manifold. Accordingly we can consider two kinds of Riemannian manifolds depicted by the metrics

$$
\begin{aligned}
& d s_{\mathbb{A}}^{2}=e^{(+) A} \otimes e^{(+) A}, \\
& d s_{\mathbb{B}}^{2}=e^{(-) A} \otimes(-) A
\end{aligned}
$$

where $\mathbb{A}$ and $\mathbb{B}$ refer to their chirality class. Each of the metrics defines their own spin connections $\omega_{A B}^{( \pm)}=\omega_{A B}^{( \pm)}\left(e^{( \pm)}\right)$ through the torsion-free condition (34). Generally speaking, the six-dimensional spin manifolds described by $\mathbb{A}$ and $\mathbb{B}$ metrics (116) are independent of each other, so the variety is simply doubled due to the Hodge duality on $\Lambda^{*} M$.

The spin connections can take arbitrary values as far as they satisfy the integrability condition (35). Their symmetry properties can be characterized by decomposing them into the irreducible subspaces under $S O(6)$ group:

$$
\omega_{A B C} \in \mathbf{6} \otimes \mathbf{1 5}=\square \otimes \boxminus=\boxminus \oplus \boxminus=20 \oplus 70
$$

where $\theta=20$ is a completely antisymmetric part of spin connections defined by $\omega_{[A B C]}=(1 / 3)\left(\omega_{A B C}+\omega_{B C A}+\omega_{C A B}\right)$. In six dimensions, the spin connections $\omega_{[A B C]}$ may be further decomposed into (imaginary) self-dual (sd) and anti-selfdual (asd) parts; that is,

$$
\omega_{[A B C]}=\left(\omega_{[A B C]}^{s d} \in \mathbf{1 0}\right) \oplus\left(\omega_{[A B C]}^{a s d} \in \mathbf{1 0}\right) .
$$

The above decomposition may be shaky because $\theta=\mathbf{2 0}$ is already an irreducible representation of $S O(6)$. It is just for a heuristic comparison with the irreducible $S U(4)$ representation. Note that $\mathbf{6}$ is coming from the antisymmetric tensor in $\mathbf{4} \times \mathbf{4}$ in (47) or $\overline{\mathbf{4}} \times \overline{\mathbf{4}}$ in (48). Thus, under $S U(4)$ group, one can instead get the following decomposition of the spin connections [20]:

$$
\omega_{A B C} \in 6 \otimes 15=\boxminus \otimes \boxminus=(6=\bigoplus) \oplus(10=\bar{\theta}) \oplus(\overline{10}=\boxplus) \oplus(64=\boxplus) .
$$

Hence notice that the irreducible representation of $S U(4)$ for spin connections is more refined than the irreducible spinor representation of $\mathrm{SO}(6)$.
Given a metric $d s^{2}=e^{A} \otimes e^{A}$, one can determine the spin connection $\omega_{A B}$ using the torsion free condition, $T^{A}=$ $d e^{A}+\omega^{A}{ }_{B} \wedge e^{B}=0$. Because we are dictating an irreducible 
spinor representation of local Lorentz symmetry for the identification (17), it is necessary to specify which representation is chosen to embed the spin connection $\omega_{A B}$. One can equally choose either the positive or negative chiral representation. This situation may be familiar with a supersymmetric solution in supergravity. To be specific, consider the supersymmetry transformation of six-dimensional gravitino $\Psi_{M}$ given by $\delta \Psi_{M}=D_{M} \eta$, where a Dirac operator $D_{M}=\partial_{M}+\omega_{M}$ acts on a chiral spinor $\eta$. Then a background geometry obeying $\delta \Psi_{M}=$ $D_{M} \eta=0$ must satisfy a well-known condition $\left[D_{M}, D_{N}\right] \eta=$ $(1 / 2) R_{M N P Q} J^{P Q} \eta=0$. In this case the representation is determined by an unbroken supersymmetry generated by the chiral spinor $\eta$. Hence the corresponding $S U(4)$ gauge fields are also identified according to the map (61), depending on the chiral representation chosen by the supersymmetric background geometry. Whenever a metric is known in a specific chiral representation, one can determine the coefficients $f_{(++)}^{a b}$ in (64) or $f_{(--)}^{a b}$ in (65) through the explicit calculation of Riemann curvature tensors. Since the geometric structures described by the data $\left(e^{(+) A}, \omega_{A B}^{(+)}\right)$and $\left(e^{(-) A}, \omega_{A B}^{(-)}\right)$ are completely independent of each other, one can attribute them to two different Riemannian manifolds.

So let us denote the geometric structures $\left(e^{(+) A}, \omega_{A B}^{(+)}\right)$ and $\left(e^{(-) A}, \omega_{A B}^{(-)}\right)$by $\mathbb{A}$ and $\mathbb{B}$, respectively, according to (31). Suppose that the geometric structures $(\mathbb{A}, \mathbb{B})$ describe a pair of six-dimensional spin manifolds $\left(M_{+}, M_{-}\right)$. Since each manifold can be described by either $\mathbb{A}$-type or $\mathbb{B}$ type, the geometric data for the pair are given by either $\left(M_{+}(\mathbb{A}), M_{-}(\mathbb{B})\right)$ or $\left(M_{+}(\mathbb{B}), M_{-}(\mathbb{A})\right)$. This pairing has shown up in Figure 1. In general, the manifolds $\left(M_{+}, M_{-}\right)$in the pair are assumed to be different even topologically. Given their metrics for the pair, one can determine the coefficients $\left(f_{(++)}^{a b}, f_{(--)}^{a b}\right)$ in (64) and (65). Since the pair consist of independent manifolds, it is possible to arrange the pair such that the coefficients $\left(f_{(++)}^{a b}, f_{(--)}^{a b}\right)$ obey some relation, for example, (1). To be specific, let us choose the embedding $\left(M_{+}(\mathbb{A}), M_{-}(\mathbb{B})\right)$. Thus one CY manifold $M_{+}=M$ is described by the metric $d s_{\mathbb{A}}^{2}=e^{(+) A} \otimes e^{(+) A}$ of type $\mathbb{A}$ while the other CY manifold $M_{-}=\widetilde{M}$ is described by the metric $d s_{\mathbb{B}}^{2}=$ $e^{(-) A} \otimes e^{(-) A}$ of type $\mathbb{B}$. The Euler characteristic $\chi(M)$ of a six-dimensional Riemannian manifold $M$ is defined by

$$
\chi(M)=\sum_{r=0}^{6}(-)^{r} b_{r}
$$

where $b_{r}=\sum_{p+q=r} h^{p, q}(M)$ is the $r$ th Betti number. A mirror pair of CY manifolds $(M, \widetilde{M})$ obeys the property $h^{p, q}(M)=$ $h^{3-p, q}(\widetilde{M})$. This property leads to an important result that the Euler characteristic of the mirror manifold $\widetilde{M}$ has an opposite sign; that is, $\chi(\widetilde{M})=-\chi(M)$. Thus the mirror symmetry implies that every CY manifold has a partner with an opposite Euler characteristic. We will use this fact to identify a mirror CY manifold.

Recall that the CY manifold $M$ is of type $A$ while the other CY manifold $\widetilde{M}$ is of type $\mathbb{B}$. Thus the spin connection of $M(\widetilde{M})$ acts on the spinor vector space $S_{+}=4\left(S_{-}=\right.$ $\overline{4})$ of positive (negative) chirality. Since classes $\mathbb{A}$ and $\mathbb{B}$ are completely independent and defined in the different vector spaces, one can choose the pair $(M, \widetilde{M})$ such that their Euler characteristics satisfy the relation $\chi(M)=-\chi(\widetilde{M})$ and so the mirror relation (1). Let us explain why this is possible.

Every complex vector bundle $E$ of rank $n$ has an underlying real vector bundle $E_{\mathbb{R}}$ of rank $2 n$, obtained by discarding the complex structure on each fiber. Then the top Chern class of a complex vector bundle $E$ is the Euler class of its realization $[21]$ :

$$
c_{n}(E)=e\left(E_{\mathbb{R}}\right),
$$

where $n=\operatorname{rank} E$. Therefore, the Euler characteristic $\chi(M)$ of $M$ for a tangent bundle $E_{\mathbb{R}}=T M$ is given by the integral of the top Chern class:

$$
\chi(M)=\int_{M} c_{n}(E)
$$

Recall that if $E$ is a complex vector bundle, then there exists a dual or conjugate bundle $\bar{E}$ with an opposite complex structure whose $j$ th Chern class is given by $[9,21]$

$$
c_{j}(\bar{E})=(-1)^{j} c_{j}(E) .
$$

The Euler characteristic $\chi(M)$ for a six-dimensional Riemannian manifold $M$ is given by

$$
\begin{aligned}
& \chi(M) \equiv-\frac{1}{2^{7} \cdot 3 \pi^{3}} \int_{M} \varepsilon^{A_{1} A_{2} \cdots A_{6}} R_{A_{1} A_{2}} \wedge R_{A_{3} A_{4}} \wedge R_{A_{5} A_{6}} \\
& =-\frac{1}{2^{10} \cdot 3 \pi^{3}} \\
& \quad \cdot \int_{M} d^{6} x \varepsilon^{M_{1} M_{2} \cdots M_{6}} \varepsilon^{A_{1} A_{2} \cdots A_{6}} R_{M_{1} M_{2} A_{1} A_{2}} R_{M_{3} M_{4} A_{3} A_{4}} R_{M_{5} M_{6} A_{5} A_{6}} .
\end{aligned}
$$

On one hand, for type $\mathbb{A}$ in (57) where $R_{A B}^{(+)}=F^{(+) a} \eta_{A B}^{a}$, it is given by

$$
\begin{aligned}
& \chi_{+}(M) \\
&=-\frac{1}{2^{7} \cdot 3 \pi^{3}} \int_{M} \varepsilon^{A_{1} A_{2} \cdots A_{6}} R_{A_{1} A_{2}}^{(+)} \wedge R_{A_{3} A_{4}}^{(+)} \wedge R_{A_{5} A_{6}}^{(+)} \\
&=-\frac{1}{2^{10} \cdot 3 \pi^{3}} \int_{M}\left(\varepsilon^{A_{1} A_{2} \cdots A_{6}} \eta_{A_{1} A_{2}}^{a} \eta_{A_{3} A_{4}}^{b} \eta_{A_{5} A_{6}}^{c}\right) F^{(+) a} \\
& \wedge F^{(+) b} \wedge F^{(+) c} \\
&=-\frac{1}{96 \pi^{3}} \int_{M} d^{a b c} F^{(+) a} \wedge F^{(+) b} \wedge F^{(+) c}
\end{aligned}
$$

where (B.9) was used. On the other hand, for type $\mathbb{B}$ in (58) where $R_{A B}^{(-)}=F^{(-) a} \bar{\eta}_{A B}^{a}$, the Euler characteristic (124) can be written as

$$
\begin{aligned}
\chi_{-} & (\widetilde{M}) \\
& =\frac{1}{2^{7} \cdot 3 \pi^{3}} \int_{\widetilde{M}} \varepsilon^{A_{1} A_{2} \cdots A_{6}} R_{A_{1} A_{2}}^{(-)} \wedge R_{A_{3} A_{4}}^{(-)} \wedge R_{A_{5} A_{6}}^{(-)} \\
& =\frac{1}{96 \pi^{3}} \int_{\widetilde{M}} d^{a b c} F^{(-) a} \wedge F^{(-) b} \wedge F^{(-) c}
\end{aligned}
$$


where (B.10) was used (in order to define the Euler characteristic for type $\mathbb{B}$, it is considered that the flip of chirality corresponds to the parity transformation (see Appendix A) and so the orientation reversal. That is the reason for the sign flip of $\chi_{-}(\widetilde{M})$. But there is some ambiguity for the choice of sign because the six-dimensional Euler characteristic needs not be positive unlike the four-dimensional case. This sign ambiguity is insignificant since it can be compensated with the redefinition $R_{A B}^{(-)} \rightarrow-R_{A B}^{(-)}$. Hence one may keep the same sign convention for $\chi_{+}(M)$ and $\chi_{-}(\widetilde{M})$. In any case the mirror pair $(M, \widetilde{M})$ will be defined by the condition $\chi_{+}(M)=$ $-\chi_{-}(\widetilde{M})$. The doubling of geometric variety guarantees the freedom to arrange a mirror pair $(M, \widetilde{M})$ to satisfy the relation $\left.\chi_{+}(M)=-\chi_{-}(\widetilde{M})\right)$. It is straightforward to represent the above Euler characteristics in terms of the chiral bases (62) and (63). For type $\mathbb{A}$ where $F^{(+) a}=f_{(++)}^{a b} J_{+}^{b}, \chi_{+}(M)$ using the identity (83) reads as

$$
\begin{aligned}
\chi_{+} & (M) \\
& =-\frac{1}{192 \pi^{3}} \int_{M} d^{6} x \sqrt{g^{(+)}} d^{a b c} d^{d e f} f_{(++)}^{a d} f_{(++)}^{b e} f_{(++)}^{c f} .
\end{aligned}
$$

Similarly, $\chi_{-}(\widetilde{M})$ for type $\mathbb{B}$ where $F^{(-) a}=f_{(--)}^{a b} J_{-}^{b}$ can be written as

$$
\begin{aligned}
\chi_{-} & (\widetilde{M}) \\
& =\frac{1}{192 \pi^{3}} \int_{\widetilde{M}} d^{6} x \sqrt{g^{(-)}} d^{a b c} d^{d e f} f_{(--)}^{a d} f_{(--)}^{b e} f_{(--)}^{c f} .
\end{aligned}
$$

Recall that two irreducible spinor representations of Spin(6) can be identified with the fundamental and antifundamental representations of $S U(4)$. By choosing a complex structure, the Spin(6) tangent bundle TM reduces to a $U(3)$ vector bundle $E$. In order to utilize the relation (122), let us consider the $U(3) \subset S U(4)$ subbundle $E$ such that $T M \otimes \mathbb{C}=$ $E \oplus \bar{E}$. Note that $U(3)$ does not mix an underlying complex structure. Thus we embed class $A$ into the $U(3)$ vector bundle $E$ over $M$. Similarly, by considering the complexification $T \widetilde{M} \otimes \mathbb{C}=F \oplus \bar{F}$, class $\mathbb{B}$ is embedded into the $U(3)$ vector bundle $\bar{F}$ over $\widetilde{M}$. It is important to recall that the curvature coefficients $f_{(++)}^{a b}$ and $f_{(--)}^{a b}$ are determined by completely independent metrics $g^{(+)}$on $M$ and $g^{(-)}$on $\widetilde{M}$, respectively. Therefore, it is always possible to find a pair $(M, \widetilde{M})$ such that the Euler characteristics (127) for type $\mathbb{A}$ and (128) for type $\mathbb{B}$ have a precisely opposite sign; that is, $\chi_{+}(M)=-\chi_{-}(\widetilde{M})$. For a CY manifold $M$ whose holonomy is $S U(3)$, the structure constants $d^{a b c}$ take values only in the $s u(3) \subset u(3)$ Lie algebra. In this case, the Euler characteristic $\chi(M)$ is given by [1]

$$
\chi(M)=2\left(h^{1,1}(M)-h^{2,1}(M)\right) .
$$

Considering the definition of the Hodge number $h^{p, q}(M)=$ $\operatorname{dim} H^{p, q}(M) \geq 0$, the sign flip of the Euler characteristics, $\chi_{+}(M)=-\chi_{-}(\widetilde{M})$, can be explained if the pair $(M, \widetilde{M})$ satisfy the mirror relation

$$
\begin{aligned}
& h^{1,1}(\mathbb{A})=h^{2,1}(\mathbb{B}), \\
& h^{1,1}(\mathbb{B})=h^{2,1}(\mathbb{A}) .
\end{aligned}
$$

Indeed the mirror relation (130) is the only way to explain the sign flip of the Euler characteristic.

The mirror symmetry (130) can be further clarified by using the fact that the Euler characteristic $\chi(M)$ of a spin manifold $M$ is related to the index of the Dirac operator on $M$ [2]. Denote the Dirac index for fermion fields in a representation $R$ by index $(R)$. The Euler characteristic $\chi(M)$ is then given by

$$
\chi(M)=\operatorname{index}(R)-\operatorname{index}(\bar{R}),
$$

where $\bar{R}$ is the complex conjugate representation of $R$. Let 4 be the fundamental representation of $S U(4)$ and $\overline{4}$ its complex conjugate, that is, the antifundamental representation. Then index $(\overline{4})=-$ index $(4)$ since in six dimensions the complex conjugation exchanges positive and negative chirality zero modes while also exchanging 4 and $\overline{4}$. Under the $S U(3)$ representation, $\mathbf{4}=\mathbf{1} \oplus \mathbf{3}$ and $\overline{\mathbf{4}}=\overline{\mathbf{1}} \oplus \overline{\mathbf{3}}$, where $\operatorname{index}(\mathbf{1})=$ index $(\overline{\mathbf{1}})=0$, so the Euler characteristic (131) is given by [2]

$$
\begin{aligned}
\chi(M) & =\operatorname{index}(\mathbf{4})-\operatorname{index}(\overline{4})=2 \operatorname{index}(\mathbf{4}) \\
& =\operatorname{index}(\mathbf{3})-\operatorname{index}(\overline{3})=2 \operatorname{index}(\mathbf{3})
\end{aligned}
$$

Then the identity (132) immediately implies the relation $\chi_{+}(M)=-\chi_{-}(\widetilde{M})$ for a pair of spin manifolds embedded in the opposite chirality representations $\mathbf{4}$ and $\overline{\mathbf{4}}$ (or $\mathbf{3}$ and $\overline{\mathbf{3}}$ for CY manifolds). This result is consistent with the mirror symmetry (130) since $\mathbb{A} \cong \mathbf{3}$ and $\mathbb{B} \cong \overline{\mathbf{3}}$.

\section{Mirror Symmetry from Gauge Theory}

In Section 3, the six-dimensional Euclidean gravity has been formulated as $S U(4) \cong S p i n(6)$ Yang-Mills gauge theory. It was shown that a Kähler manifold is described by the reduced $U(3) \subset S U(4)$ gauge symmetry. After imposing the Ricciflat condition on the Kähler manifold, the gauge group in the Yang-Mills theory is further reduced to $S U(3)$. After all, a $C Y$ manifold $M$ from the gauge theory point of view can be described by $S U$ (3) connections supported on $M$ satisfying the HYM equations. And the mirror symmetry says that a CY manifold has a mirror pair satisfying the relation (1). Therefore, there must be a corresponding HYM instanton which can be derived from a mirror CY manifold obeying the mirror relation (130). In this section we will identify the mirror HYM instanton from the gauge theory approach and then clarify the mirror symmetry between CY manifolds from the gauge theory formulation.

Suppose that the metric of a six-dimensional Riemannian manifold $M$ is given by

$$
d s^{2}=g_{M N}(x) d x^{M} d x^{N} .
$$

Let $\pi: E \rightarrow M$ be an $S U(4)$ bundle over $M$ whose curvature is defined by

$$
\begin{aligned}
F & =d A+A \wedge A \\
& =\frac{1}{2}\left(\partial_{M} A_{N}-\partial_{N} A_{M}+\left[A_{M}, A_{N}\right]\right) d x^{M} \wedge d x^{N},
\end{aligned}
$$


where $A=A_{M}^{a}(x) T^{a} d x^{M}$ is a connection one-form vector space on the vector bundle $E$. The generators $T^{a}$ of $s u(4)$ Lie algebra satisfy the commutation relation (16) with normalization $\operatorname{Tr} T^{a} T^{b}=-(1 / 2) \delta^{a b}$. Consider the $S U$ (4) Yang-Mills gauge theory defined on the Riemannian manifold $M$ with the metric (133) whose action is given by

$$
S_{Y M}=-\frac{1}{2 g_{Y M}^{2}} \int_{M} d^{6} x \sqrt{g} g^{M P} g^{N Q} \operatorname{Tr} F_{M N} F_{P Q}
$$

Using the projection operator (71) and the identity (75), it is easy to derive the following formula:

$$
\begin{aligned}
\left(P_{ \pm} F\right)^{2}= & \left(P_{ \pm}^{A_{1} B_{1} A_{2} B_{2}} F_{A_{2} B_{2}}\right)\left(P_{ \pm}^{A_{1} B_{1} A_{3} B_{3}} F_{A_{3} B_{3}}\right) \\
= & \frac{1}{4}\left(F_{A_{1} B_{1}} \pm \frac{1}{4} \varepsilon^{A_{1} B_{1} A_{2} B_{2} A_{3} B_{3}} F_{A_{2} B_{2}} I_{A_{3} B_{3}}\right)^{2} \\
= & \frac{1}{2} F_{A B} F^{A B} \pm \frac{1}{8} \varepsilon^{A B C D E F} F_{A B} F_{C D} I_{E F} \\
& +\frac{1}{8}\left(I_{A B} F^{A B}\right)^{2} \\
= & P_{ \pm}^{A B C D} F_{A B} F_{C D}+\frac{1}{8}\left(I_{A B} F^{A B}\right)^{2} .
\end{aligned}
$$

One can rewrite the action (135) using the above identity as

$$
\begin{aligned}
S_{Y M} & =-\frac{1}{4 g_{Y M}^{2}} \\
\cdot & \int_{M} d^{6} x \sqrt{g} \operatorname{Tr}\left[\left(F_{A B} \pm \frac{1}{4} \varepsilon^{A B C D E F} F_{C D} I_{E F}\right)^{2}\right. \\
& \left.-\frac{1}{2}\left(I_{A B} F^{A B}\right)^{2} \mp \frac{1}{2} \varepsilon^{A B C D E F} F_{A B} F_{C D} I_{E F}\right] .
\end{aligned}
$$

The above action can be written in a more compact form as

$$
\begin{aligned}
S_{Y M} & =-\frac{1}{4 g_{Y M}^{2}} \int_{M} d^{6} x \sqrt{g} \\
\cdot & \operatorname{Tr}\left[\left(F_{A B} \pm *(F \wedge \Omega)_{A B}\right)^{2}-\frac{1}{2}\left(I_{A B} F^{A B}\right)^{2}\right] \\
\pm & \frac{1}{g_{Y M}^{2}} \int_{M} d^{6} x \operatorname{Tr} F \wedge F \wedge \Omega,
\end{aligned}
$$

where $\Omega$ is the two-form vector space of rank 6 defined by (84).

Using the fact

$$
\operatorname{Tr} F \wedge F=d \operatorname{Tr}\left(A \wedge F-\frac{1}{3} A \wedge A \wedge A\right) \equiv d K,
$$

one can see that the last term in (138) is a topological term; that is,

$$
\operatorname{Tr} F \wedge F \wedge \Omega=d(K \wedge \Omega)
$$

if and only if the two-form $\Omega$ is closed; that is, $d \Omega=$ 0 . In other words, when $M$ is a Kähler manifold, the last term in (138) depends only on the topological class of the Kähler-form $\Omega$ and the vector bundle $E$ over $M$. Note that (138), except the second term, is very similar to the Bogomol'nyi equation for Yang-Mills instantons whose action is bounded by a topological term. Indeed we can apply the Bogomol'nyi argument to (138) thanks to the identity $(1 / 8) \mathcal{E}^{A B C D E F} I_{C D} I_{E F}=I_{A B}$. More precisely, it is easy to see that a solution obeying the $\Omega$-self-duality equations

$$
F_{A B} \pm *(F \wedge \Omega)_{A B}=0
$$

automatically satisfies the condition

$$
I_{A B} F^{A B}=0 .
$$

Therefore, the minimum action can be achieved by the configuration satisfying (141) and is given by the last term-the topological term-in (138). Note that we have already encountered the above self-duality equations in (91) and (92). They can be summarized as the so-called DUY equations $[17,18]$

$$
\begin{aligned}
F^{(2,0)} & =F^{(0,2)}=0, \\
F \wedge \Omega^{2} & =0 .
\end{aligned}
$$

The first equation states that the $S U(4)$ gauge field is a connection on a holomorphic vector bundle and the last condition corresponds to the stability of the holomorphic vector bundle in algebraic geometry. It is straightforward to show $[1,22]$ that a solution of the self-duality equations (141) automatically satisfies the Yang-Mills equations of motion

$$
g^{M N} D_{M} F_{N P}=0
$$

on a Kähler manifold.

Let us analyze the HYM equations (141). We observed in Section 3 that the 't Hooft symbols in (59) realizes the isomorphism between irreducible spin(6) Lorentz algebra and $s u(4)$ Lie algebra and provides a complete basis of two forms in $\Omega_{+}^{2}(M)$. For instance, one may expand the $S U(4)$ field strengths $F_{A B}^{a}(a=1, \ldots, 15)$ using the basis (59) like either (62) or (63). A question is how to realize the doubling of $\mathrm{CY}$ manifolds from the $S U(4)$ gauge theory approach. The crux for this question is that the $N$-dimensional fundamental representation of $S U(N)$ for $N$ greater than two is a complex representation, whose complex conjugate is often called the antifundamental representation. And the complex conjugate representation $\overline{\mathbf{N}}$ is an inequivalent representation different from the original one $\mathbf{N}$. In particular, the positive and negative chirality representations of $\operatorname{Spin}(6) \cong S U(4)$ coincide with the fundamental (4) and the antifundamental $(\overline{4})$ representations of $S U(4)$. Therefore, we have a freedom to embed the solutions of Yang-Mills gauge theory in a specific representation. This freedom is basically related to the existence of two independent bases of two forms, $\eta_{A B}^{a}$ and $\bar{\eta}_{A B}^{a}$, according to the isomorphism (B) and (C).

Thereby we will identify the $S U(4)$ field strength $F_{A B}^{a}$ in the fundamental representation 4 with type $\mathbb{A}$ in (62) and in the antifundamental representation $\overline{4}$ with type $\mathbb{B}$ in (63). 
For the antifundamental representation $\overline{\mathbf{4}}$, the Lie algebra generators are given by $\left(T^{a}\right)^{*}=-(i / 2) \lambda_{a}^{*}$ and they obey the same Lie algebra as $T^{a}$ :

$$
\left[\left(T^{a}\right)^{*},\left(T^{b}\right)^{*}\right]=-f^{a b c}\left(T^{c}\right)^{*} .
$$

But one can see from (A.9) that the symmetric structure constants have an opposite sign; that is,

$$
\begin{aligned}
\operatorname{Tr}\left\{T^{a}, T^{b}\right\} T^{c} & =-\frac{i}{2} d^{a b c}, \\
\operatorname{Tr}\left\{\left(T^{a}\right)^{*},\left(T^{b}\right)^{*}\right\}\left(T^{c}\right)^{*} & =\frac{i}{2} d^{a b c} .
\end{aligned}
$$

It turns out that this sign flip is correlated with the opposite sign in (A.5). According to the tensor product (67), one can decompose the coefficients $f_{( \pm \pm)}^{a b}$ into a symmetric part and an antisymmetric part

$$
f_{( \pm \pm)}^{a b}=f_{( \pm \pm)}^{(a b)}+f_{( \pm \pm)}^{[a b]}
$$

Although it is not necessary to impose the symmetry property (68) for a general vector bundle $\pi: E \rightarrow M$, we will impose the symmetric prescription, that is, $f_{( \pm \pm)}^{[a b]}=0$, because we are interested in the gauge theory formulation of six-dimensional Riemannian manifolds where $E=T M$ and the bundle connections are identified with spin connections (of course, the symmetric condition (149) greatly reduces the number of field strengths $(225 \rightarrow 120)$. According to the relation (B.5) for the tangent bundle $E=T M$, it is easy to derive the identities $\left[F_{A B}^{( \pm)}, J_{ \pm}^{A B}\right]=0$ and $(1 / 4)\left\{F_{A B}^{( \pm)}, J_{ \pm}^{A B}\right\}=$ $\left.-(1 / 8) f_{( \pm \pm)}^{a b} \delta^{a b}+(i / 2) d^{a b c} f_{( \pm \pm)}^{a b} T_{ \pm}^{c}\right)$. Then,

$$
F_{A B}^{( \pm) a}=f_{( \pm \pm)}^{a b} \eta_{A B}^{( \pm) b}
$$

where we have omitted the symmetrization symbol with respect to $a \leftrightarrow b$ for brevity.

Now let us consider the HYM equations on a Kähler manifold $M$. Recall that the HYM equation (141) can be resolved by decomposing the Yang-Mills field strengths (149) into the eigenspaces of the Hodge operator (85). And we showed that the decomposition (86) is equivalent to the branching (98) of $S U(4)$ under the $U(3)$ subgroup since the $S U(4)$ gauge group is reduced to $U(3)$ by the background Kähler class $\Omega$.(It might be obvious from the expansion (149) which intertwines the $S U(4)$ index $a$ and $S O(6)$ indices $A, B$. Note that the different choice of background Kähler classes can be parameterized by the homogeneous space $S U(4) / U(3)=\mathbb{C} P^{3}$. Also (B.17) implies that the space $\mathbb{C} P^{3}=S U(4) / U(3)$ can be identified with the space of complex structure deformations [9]. This coincidence might presage the mirror symmetry.) Therefore, the Yang-Mills field strengths obeying (141) take values in $u(3)$ Lie algebra; that is, $a, b$ run over $3,6,7,8,11,12,13,14,15$ for the fundamental representation 4 and 3, 4, 5, 8, 9, 10, 13, 14, 15 for the antifundamental representation $\overline{4}$. To be specific, $\eta_{A B}^{a} \in\left\{l_{A B}^{(+) \widehat{a}}, n_{A B}^{(+) 0}\right\}$ for 4 and $\bar{\eta}_{A B}^{a} \in\left\{l_{A B}^{(-) \widehat{a}}, n_{A B}^{(-) 0}\right\}$ for $\overline{\mathbf{4}}$ with the 't Hooft symbols $l_{A B}^{( \pm) \widehat{a}}$ and $n_{A B}^{( \pm) 0}$ defined in Section 3. As the background Kähler class $\Omega$ determines a particular $U(3) \subset S U(4)$ subgroup and 4 and $\overline{4}$ belong to two different representations, the Kähler classes in the representations $\mathbf{4}$ and $\overline{4}$ should be attributed to different Kähler manifolds.

Hence let us consider the $S U(4)$ gauge theory defined on two different Kähler manifolds $M$ and $\widetilde{M}$ whose background Kähler classes are, respectively, given by

$$
\begin{aligned}
& \Omega_{+}=n_{A B}^{(+) 0} e^{(+) A} \wedge e^{(+) B}, \\
& \Omega_{-}=n_{A B}^{(-) 0} e^{(-) A} \wedge e^{(-) B} .
\end{aligned}
$$

Given a fixed Kähler class, the HYM equations will be solved by $U(3)$ connections. The stability equation (142) for each case is then reduced to the following equations:

$$
\begin{aligned}
& I_{A B} F_{A B}^{(+) a}=f_{(++)}^{a b} \eta_{A B}^{b} I_{A B}=0 \Longleftrightarrow \\
& f_{(++)}^{3 a}+\frac{1}{\sqrt{3}} f_{(++)}^{8 a}+\frac{1}{\sqrt{6}} f_{(++)}^{15 a}=0, \\
& I_{A B} F_{A B}^{(-) a}=f_{(--)}^{a b} \bar{\eta}_{A B}^{b} I_{A B}=0 \Longleftrightarrow \\
& f_{(--)}^{3 a}-\frac{1}{\sqrt{3}} f_{(--)}^{8 a}-\frac{1}{\sqrt{6}} f_{(--)}^{15 a}=0 .
\end{aligned}
$$

By applying the exactly same argument as Section 3, one can conclude that the above equations are equivalent to the vanishing of the first Chern-class; that is,

$$
\begin{aligned}
& F^{(+) 0}=d A^{(+) 0}=0, \\
& F^{(-) 0}=d A^{(-) 0}=0,
\end{aligned}
$$

where the $U(1)$ gauge fields $A^{( \pm) 0}$ are defined by (107) and (112). One can also see from (91) that the $S U(3)$ basis $\left\{l_{A B}^{( \pm) \widehat{a}}\right\}$ definitely picks up the +-sign in (141) and its solution is given by

$$
F_{A B}^{( \pm) \widehat{a}}=f_{( \pm \pm)}^{\widehat{a} \hat{b}} l_{A B}^{( \pm) \widehat{b}}, \quad \widehat{a}, \widehat{b}=1, \ldots, 8
$$

Consequently we found that the HYM instanton inherited from a CY manifold is described by the $S U(3)$ connections with the trivial first Chern class. This means that the tangent bundle TM of a CY manifold $M$ gives rise to $S U(3)$ connections in a stable holomorphic vector bundle [1]. This is exactly the statement of the DUY theorem $[17,18]$ for a particular case of the vector bundle $E=T M$ over a CY manifold $M$. Now it becomes clear what is the mirror relation for the HYM instantons. The mirror symmetry of CY manifolds can be understood as the relationship between two kinds of HYM instantons in SU (4) gauge theory embedded in the fundamental representation $\mathbf{4}$ and the antifundamental representation $\overline{4}$. Each representation has its own cohomology classes, taking values in the holomorphic vector bundles $E$ over $M$ and $\bar{F}$ over $\widetilde{M}$. It should be remarked that we intend to construct the complex vector bundles $E$ and $\bar{F}$ via the tangent bundles $T M \otimes \mathbb{C}=E \oplus \bar{E}$ and $T \widetilde{M} \otimes \mathbb{C}=F \oplus \bar{F}$, so the vector bundles $E$ and $\bar{F}$ are independent of each other. Since 
the Kähler class $\Omega_{ \pm}$reduces the gauge group to $U(3)$, the underlying complex structures are not mixed under gauge transformations.

Since we want to understand the mirror symmetry between CY manifolds in terms of $S U(4)$ gauge theory, it will be useful to calculate the Chern classes of the vector bundle to elucidate the mirror symmetry between HYM instantons. It was already shown that the first Chern class $c_{1}(E)$ of the holomorphic vector bundle satisfying (141) is trivial; that is, $c_{1}(E)=0$. Also we have shown that the last term in (138) is a topological invariant which contains the second Chern class $c_{2}(E)$. After using (141), one can derive the inequality

$$
\frac{1}{8 \pi^{2}} \int_{M_{ \pm}} \operatorname{Tr} F^{( \pm)} \wedge F^{( \pm)} \wedge \Omega_{ \pm} \geq 0
$$

where

$$
c_{2}\left(V_{ \pm}\right)=\frac{1}{8 \pi^{2}} \operatorname{Tr} F^{( \pm)} \wedge F^{( \pm)}
$$

is the second Chern class of a complex vector bundle $V_{+}=E$ over $M_{+}=M$ or $V_{-}=\bar{F}$ over $M_{-}=\widetilde{M}$. This is known as the Bogomolov inequality $[22,23]$, which is true for all stable bundles with $c_{1}\left(V_{ \pm}\right)=0$. Using the identification in (57) and (58), one may translate the above inequality into the one in gravity theory

$$
-\frac{1}{16 \pi^{2}} \int_{M_{ \pm}} R_{A B}^{( \pm)} \wedge R_{A B}^{( \pm)} \wedge \Omega_{ \pm} \geq 0
$$

Finally, according to formula (122), we calculate the integral of the third Chern class $c_{3}\left(V_{ \pm}\right)$given by

$$
\begin{aligned}
& \chi_{+}(E)=-\frac{i}{24 \pi^{3}} \int_{M} \operatorname{Tr} F^{(+)} \wedge F^{(+)} \wedge F^{(+)} \\
& =-\frac{1}{96 \pi^{3}} \int_{M} d^{a b c} F^{(+) a} \wedge F^{(+) b} \wedge F^{(+) c} \\
& =-\frac{1}{192 \pi^{3}} \int_{M} d^{6} x \sqrt{g^{(+)}} d^{a b c} d^{d e f} f_{(++)}^{a d} f_{(++)}^{b e} f_{(++)}^{c f}, \\
& \chi_{-}(\bar{F})=-\frac{i}{24 \pi^{3}} \int_{\widetilde{M}} \operatorname{Tr} F^{(-)} \wedge F^{(-)} \wedge F^{(-)} \\
& =\frac{1}{96 \pi^{3}} \int_{\widetilde{M}} d^{a b c} F^{(-) a} \wedge F^{(-) b} \wedge F^{(-) c} \\
& =\frac{1}{192 \pi^{3}} \int_{\widetilde{M}} d^{6} x \sqrt{g^{(-)}} d^{a b c} d^{d e f} f_{(--)}^{a d} f_{(--)}^{b e} f_{(--)}^{c f} .
\end{aligned}
$$

It might be remarked that the relative $\operatorname{sign} c_{3}(\bar{F})=-c_{3}(F)$ for the third Chern classes of a complex vector bundle $F$ and its conjugate bundle $\bar{F}$ arises from the property (147). Since the complex vector bundles $E$ and $\bar{F}$ are independently defined over two different Kähler manifolds $M$ and $\widetilde{M}$, respectively, the expansion coefficients $f_{( \pm \pm)}^{a b}$ in (149) will also be separately determined by them. Hence it should be possible to construct a pair of complex vector bundles $(E \rightarrow M, \bar{F} \rightarrow \widetilde{M})$ such that $\chi_{+}(E)=-\chi_{-}(\bar{F})$. One may notice that the sign flip in the Euler characteristic is also consistent with the general result
(122). In consequence, the above Euler characteristics correctly reproduce (127) and (128) for the CY manifold $M$ and its mirror manifold $\widetilde{M}$. This constitutes a gauge theory formulation of mirror symmetry.

In conclusion we have confirmed the picture depicted in Figure 1 that the mirror symmetry between CY manifolds can be understood as the mirror pair of HYM instantons in the fundamental representations 3 and $\overline{3}$ of $S U(3)$ gauge connections. Since the existence of two different fundamental representations of $S U(4) \cong \operatorname{Spin}(6)$ is related to the doubling of the vector space in (6) according to the isomorphism (4), we see that the mirror symmetry of CY manifolds and HYM instantons originates from the Hodge duality in the vector space $\Lambda^{*} M$.

\section{Discussion}

The physics on a curved spacetime becomes more transparent when expressed in a locally inertial frame and it is even indispensable when one wants to couple spinors to gravity since spinors in $d$-dimensions form a representation of $\operatorname{Spin}(d)$ Lorentz group rather than $G L(d, \mathbb{R})$. It is also required to take an irreducible spinor representation of the Lorentz symmetry. Then one can apply the elementary propositions $(\mathrm{A}, \mathrm{B}, \mathrm{C})$ in Section 1 to $d$-dimensional Riemannian manifolds to see their consequences. It is, especially, interesting to apply them to six-dimensional CY manifolds. Proposition (A) first says that Riemann curvature tensors $R_{A B C D}$ carry two kinds of indices; the first group, say $[A B]$, belongs to $\operatorname{Spin}(6)$ indices and the second group [CD] belongs to form indices in $\Omega^{2}(M)$. But proposition (C) requires that two groups must have an isomorphic structure as vector spaces. After imposing the torsion free condition that leads to the symmetry property, $R_{A B C D}=R_{C D A B}$, the vector space structure for the two groups should be even identified. For example, the irreducible spinor representation of the Lorentz group Spin(6) requires us to consider the vector spaces $\Omega^{2}(M)$ and $\Omega^{4}(M)$ on equal footing. The doubling of the vector space (6) is realized as either two independent chiral representations of the Lorentz group Spin(6) or two independent complex representations $\mathbf{4}$ and $\overline{4}$ of the gauge group $S U(4)$. We observed that the doubling of the vector spaces essentially brings about the doubling for the variety of six-dimensional spin manifolds which is responsible for the existence of the mirror symmetry between CY manifolds.

It may be worthwhile to compare the four and six dimensions in perspective. On a four-dimensional orientable manifold, the vector space of two-form $\Omega^{2}(M)$ is not doubled because the Hodge duality of a two-form vector space is again a two-form vector space. Instead the vector space $\Omega^{2}(M)$ splits canonically into two vector spaces as (7). This split is resonant with the self-duality of chiral Lorentz generators $J_{ \pm}^{A B}$ because they obey the relation

$$
J_{ \pm}^{A B}= \pm \frac{1}{2} \varepsilon^{A B C D} J_{ \pm}^{C D}
$$

Therefore, the chiral Lorentz generators $J_{ \pm}^{\mathrm{AB}}$ have three independent components only. Combining them together, they 
consist of six generators which match with the dimension of $\Omega^{2}(M)$. Applying this fact to (19), one can see that $R_{A B}^{( \pm)}$ contains $18=6 \times 3$ components and so $36=18+18$ components in total, which is the number of components of Riemann curvature tensors $R_{A B C D}$ before imposing the first Bianchi identity. This situation is different from the sixdimensional case as is evident from the comparison of (6) and (7). This difference is originated from the fact that, in six dimensions, there is another source of two-form vector space coming from the Hodge duality of four-form vector space. As a consequence, $R_{A B}^{( \pm)}$in (79) has $225=15 \times 15$ components in six dimensions and so $450=225+225$ components in total before imposing the first Bianchi identity. After imposing the first Bianchi identity in each class that totally comprises $240=120+120$ constraints, the physical curvature tensors $\left(R_{A B}^{(+)} \oplus R_{A B}^{(-)}\right)$have $210=105+105$ components in total. This doubling for the variety of $C Y$ manifolds is a core origin of the mirror symmetry between CY manifolds.

Via the gauge theory formulation of six-dimensional Euclidean gravity, we showed that HYM instantons can be constructed in two different ways by embedding them into the fundamental or antifundamental representation of $S U(4) \cong \operatorname{Spin}(6)$ gauge group. Since a CY manifold can be recast as a HYM instanton from the gauge theory point of view (see the quotation in Section 1) and the chiral representation of Spin(6) corresponds to the fundamental representation of $S U(4)$, the structure in Figure 1 has been nicely verified. After all, the mirror symmetry of CY manifolds can be understood as the existence of the mirror pair of HYM instantons by doubling the variety of six-dimensional spin manifolds according to the Hodge duality (6).

Strominger et al. recently proposed [7] that the mirror symmetry is a T-duality transformation along a dual special Lagrangian tori fibration on a mirror CY manifold. The Tduality transformation along the dual three-tori introduces a sign flip in the Euler characteristic as even and odd forms exchange their role. Note that the odd number of T-duality operations transforms type IIB string theory to type IIA string theory and vice versa. Hence types IIA and IIB CY manifolds will be mirror to each other because the six-dimensional chirality will be flipped after the T-duality and the ten-dimensional chirality is correlated with the sixdimensional one. This result implies that the mirror symmetry in string theory originates from the two different chiral representations of CY manifolds, which is consistent with our picture.

Our gauge theory formulation may be generalized to a general six-dimensional Riemannian manifold like the four-dimensional case $[12,13]$ because it is simply based on the general propositions $(\mathrm{A}, \mathrm{B}, \mathrm{C})$ in Section 1. One may consider, for example, the Strominger system [24, 25] for non-Kähler complex manifolds. The Strominger system admits a conformally balanced Hermitian form on a threedimensional compact complex manifold $M$, a nowhere vanishing holomorphic $(3,0)$-form, and a HYM connection on a vector bundle $E$ over this manifold. The consistency of the underlying physical theory imposes a constraint that the curvature forms have to satisfy the anomaly equation. As far as the non-Kähler CY manifold admits a spin structure, the gauge theory formulation for the Strominger system may be straightforward as much as we have done in this paper. Thus it may be interesting to formulate the mirror symmetry for non-Kähler manifolds from the gauge theory perspective and to generalize it to the case without spin structure, for instance, a manifold with $\mathrm{Spin}^{\mathbb{C}}$ structure only. If there is a substantial progress along this line, it will be reported elsewhere.

If we consider a CY manifold $M$ to be the HYM instanton of the tangent bundle $T M$, this instanton will have their own moduli space given by their zero modes, with the nonzero modes providing various "uplifting." Then the following questions naturally arise. How is the moduli space of HYM instantons related to the CY moduli space? Also how do the nonzero modes of the instanton solution correspond to the CY deformations?

To discuss this issue, let us consider an infinitesimal deformation of the gauge field

$$
A_{\mu}+\delta A_{\mu} .
$$

If we demand (143) for both the original gauge field and the deformation, then the deformation must satisfy $\bar{\partial} \delta A=$ 0 . This means that $\delta A \in H^{1}($ End $E)$. On a manifold of SU(3) holonomy and for the case $E=T M, H^{1}$ (End $E$ ) coincides with $H^{2,1}(M)$. Therefore, the bundle moduli (159) for the condition (143) of the holomorphic tangent bundle $T M$ correspond to the deformations of the complex structure, counted by $H^{1}(M, T M) \cong H^{2,1}(M)[2,3]$. However it is known [26] that the HYM equations (143) and (144) do not fix any of the Kähler moduli. Indeed the DUY theorem states $[17,18]$ that, for a fixed choice of Kähler moduli, there exists a solution of the HYM equations if the holomorphic vector bundle is slope-stable, which is the case for the tangent bundle. Therefore, it is not possible to extract the Kähler moduli from the bundle moduli (159). This implies that the moduli space of a CY manifold is not fully captured by the instanton moduli space even for the tangent bundle since we fix the Kähler moduli of a background CY manifold to define the HYM equations.

It is well-known that the instanton moduli space has singularities, the so-called "small instanton" singularities. Also the CY moduli space has singularities: the conifold points [3]. Thus it will be interesting to understand how these two kinds of singularities are related to each other. Our result implies that the singularities in the instanton moduli space are related to the singularities (the conifold points) of the CY moduli space because the tangent bundle $T M$ is defined by the CY manifold $M$. But the blown-up conifold singularities may arise in different ways from the instanton picture of the CY manifold since it is known [27] that there is a natural complex structure on the resolution but not a natural Kähler structure while the deformation is symplectic in a natural way but not naturally complex. Since only the complex structure deformations of the CY manifold are encoded in the bundle moduli (159), we speculate that the resolved conifold is realized from the instanton side while the deformed conifold appears in the background CY manifold. Note that the deformed conifold is mirror to the 
resolved conifold, which is related to the conifold transitions. Thus the instanton picture of CY manifolds implies that the HYM instanton for the tangent bundle TM over a deformed conifold $M$ is mirror to the HYM instanton for the tangent bundle $T \widetilde{M}$ over a resolved conifold $\widetilde{M}$. We leave this problem for the future work.

Mirror symmetry provides an isomorphism between complex geometry and symplectic geometry which relates the deformation of complex structure on the complex geometry side to the counting of pseudo-holomorphic spheres on the symplectic geometry side. A very similar picture arises in emergent gravity that isomorphically relates the deformation of symplectic structure described by a NC $U(1)$ gauge theory to the deformation of complex structure in Einstein gravity. The deformation of symplectic structure is represented by $\mathscr{F}=B+F$ where $B$ is an underlying symplectic structure on $M$ and $F=d A$ is identified with the curvature of line bundle $L \rightarrow M$. In order to allow singular $U(1)$ gauge fields such as $U(1)$ instantons, it is necessary to generalize the line bundle to a torsion free sheaf or an ideal sheaf. NCU(1) gauge fields are introduced via a local coordinate transformation $\phi \in \operatorname{Diff}(M)$ eliminating $U(1)$ gauge fields; that is, $\phi^{*}(\mathscr{F})=$ $B$, known as the Darboux theorem in symplectic geometry. It was claimed in $[28,29]$ and recently shown in $[30,31]$ that sixdimensional CY manifolds are emergent from NC Hermitian $U(1)$ instantons. Note that the NC Hermitian $U(1)$ instantons correspond to $U(1)$ connections in a stable holomorphic line bundle $L \rightarrow M$ or more generally a torsion free sheaf (an ideal sheaf) from the commutative description [32]. When we conceive the emergent CY manifolds from the mirror symmetry perspective, an interesting question is how to realize the mirror symmetry from the emergent gravity picture. It turns out [33] that the emergent gravity picture provides a very nice result for the mirror symmetry.

\section{Appendix}

\section{A. $S \operatorname{pin}(6)$ and $S U(4)$}

We consider the six-dimensional Clifford algebra with the Dirac matrices given by

$$
\Gamma^{A}=\left(\begin{array}{cc}
0 & \gamma^{A} \\
\bar{\gamma}^{A} & 0
\end{array}\right), \quad A=1, \ldots, 6
$$

where $\bar{\gamma}^{A}=\left(\gamma^{A}\right)^{\dagger}$. Thus the Dirac matrices we have taken are Hermitian; that is, $\left(\Gamma^{A}\right)^{\dagger}=\Gamma^{A}$. We choose $\left(\gamma^{i}\right)^{\dagger}=$ $\gamma^{i}(i=1, \ldots, 5)$ and $\left(\gamma^{6}\right)^{\dagger}=-\gamma^{6}$. We will use the following representation of Dirac matrices [34]:

$$
\gamma^{1}=\left(\begin{array}{cccc}
0 & 0 & 0 & -1 \\
0 & 0 & 1 & 0 \\
0 & 1 & 0 & 0 \\
-1 & 0 & 0 & 0
\end{array}\right),
$$

$$
\begin{aligned}
\gamma^{2} & =\left(\begin{array}{cccc}
0 & 0 & 0 & i \\
0 & 0 & i & 0 \\
0 & -i & 0 & 0 \\
-i & 0 & 0 & 0
\end{array}\right) \\
\gamma^{3} & =\left(\begin{array}{cccc}
0 & 0 & 1 & 0 \\
0 & 0 & 0 & 1 \\
1 & 0 & 0 & 0 \\
0 & 1 & 0 & 0
\end{array}\right) \\
\gamma^{4} & =\left(\begin{array}{cccc}
0 & 0 & -i & 0 \\
0 & 0 & 0 & i \\
i & 0 & 0 & 0 \\
0 & -i & 0 & 0
\end{array}\right), \\
\gamma^{5} & =\left(\begin{array}{cccc}
1 & 0 & 0 & 0 \\
0 & 1 & 0 & 0 \\
0 & 0 & -1 & 0 \\
0 & 0 & 0 & -1
\end{array}\right)=-\gamma^{1} \gamma^{2} \gamma^{3} \gamma^{4},
\end{aligned}
$$

satisfying the Spin(5) Clifford algebra relation

$$
\gamma^{i} \gamma^{j}+\gamma^{j} \gamma^{i}=2 \delta^{i j}, \quad i, j=1, \ldots, 5
$$

The Lorentz generators for the irreducible (chiral) spinor representation of Spin(6) are defined by

$$
J_{ \pm}^{A B} \equiv \frac{1}{2}\left(\mathbf{I}_{8} \pm \Gamma_{7}\right) J^{A B}
$$

where $\Gamma_{7}=i \Gamma^{1} \cdots \Gamma^{6}$. Note that $J_{+}^{A B}$ and $J_{-}^{A B}$ independently satisfy the Lorentz algebra (11) and commute each other; that is, $\left[J_{+}^{A B}, J_{-}^{C D}\right]=0$. They also satisfy the anticommutation relation

$$
\begin{aligned}
\left\{J_{ \pm}^{A B}, J_{ \pm}^{C D}\right\}= & -\frac{1}{2}\left(\delta^{A C} \delta^{B D}-\delta^{A D} \delta^{B C}\right) \Gamma_{ \pm} \\
& \pm \frac{i}{2} \varepsilon^{A B C D E F} J_{ \pm}^{E F} .
\end{aligned}
$$

Because the chiral matrix $\Gamma_{7}$ is given by

$$
\Gamma_{7}=\left(\begin{array}{cc}
\mathbf{I}_{4} & 0 \\
0 & -\mathbf{I}_{4}
\end{array}\right),
$$

where $\mathbf{I}_{4}$ is the $4 \times 4$ identity matrix, the generators of the chiral spinor representation in (A.4) are given by $4 \times 4$ matrices. Then the two independent chiral spinor representations of $\operatorname{Spin}(6)$ are given by

$$
\begin{aligned}
& J_{+}^{A B}=\left\{J_{+}^{i j}=\frac{1}{4}\left[\gamma^{i}, \gamma^{j}\right], J_{+}^{i 6}=\frac{i}{2} \gamma^{i}\right\}, \\
& J_{-}^{A B}=\left\{J_{-}^{i j}=\frac{1}{4}\left[\gamma^{i}, \gamma^{j}\right], J_{-}^{i 6}=-\frac{i}{2} \gamma^{i}\right\} .
\end{aligned}
$$


One can check that the generators $J_{+}^{A B}$ and $J_{-}^{A B}$ separately obey the Lorentz algebra (11).

One can exchange the positive chiral representation and the negative chiral representation by a parity transformation, which is a reflection $x^{M} \rightarrow-x^{M}$ of any one element of the fundamental six-dimensional representation of Spin(6) [1]; in our case, $x^{6} \rightarrow-x^{6}$. But they cannot be connected by any $\mathrm{SO}(6)$ rotations.

The anti-Hermitian $4 \times 4$ matrices $T^{a}=(i / 2) \lambda_{a}, a=$ $1, \ldots, 15$ with vanishing traces constitute the basis of $S U(4)$ Lie algebra. The Hermitian $4 \times 4$ matrices $\lambda_{a}$ are given by

$$
\begin{aligned}
& \lambda_{1}=\left(\begin{array}{llll}
0 & 1 & 0 & 0 \\
1 & 0 & 0 & 0 \\
0 & 0 & 0 & 0 \\
0 & 0 & 0 & 0
\end{array}\right), \\
& \lambda_{2}=\left(\begin{array}{cccc}
0 & -i & 0 & 0 \\
i & 0 & 0 & 0 \\
0 & 0 & 0 & 0 \\
0 & 0 & 0 & 0
\end{array}\right), \\
& \lambda_{3}=\left(\begin{array}{cccc}
1 & 0 & 0 & 0 \\
0 & -1 & 0 & 0 \\
0 & 0 & 0 & 0 \\
0 & 0 & 0 & 0
\end{array}\right), \\
& \lambda_{4}=\left(\begin{array}{llll}
0 & 0 & 1 & 0 \\
0 & 0 & 0 & 0 \\
1 & 0 & 0 & 0 \\
0 & 0 & 0 & 0
\end{array}\right), \\
& \lambda_{5}=\left(\begin{array}{cccc}
0 & 0 & -i & 0 \\
0 & 0 & 0 & 0 \\
i & 0 & 0 & 0 \\
0 & 0 & 0 & 0
\end{array}\right) \text {, } \\
& \lambda_{6}=\left(\begin{array}{llll}
0 & 0 & 0 & 0 \\
0 & 0 & 1 & 0 \\
0 & 1 & 0 & 0 \\
0 & 0 & 0 & 0
\end{array}\right), \\
& \lambda_{7}=\left(\begin{array}{cccc}
0 & 0 & 0 & 0 \\
0 & 0 & -i & 0 \\
0 & i & 0 & 0 \\
0 & 0 & 0 & 0
\end{array}\right),
\end{aligned}
$$

$$
\lambda_{8}=\frac{1}{\sqrt{3}}\left(\begin{array}{cccc}
1 & 0 & 0 & 0 \\
0 & 1 & 0 & 0 \\
0 & 0 & -2 & 0 \\
0 & 0 & 0 & 0
\end{array}\right)
$$$$
\lambda_{9}=\left(\begin{array}{llll}
0 & 0 & 0 & 1 \\
0 & 0 & 0 & 0 \\
0 & 0 & 0 & 0 \\
1 & 0 & 0 & 0
\end{array}\right),
$$$$
\lambda_{10}=\left(\begin{array}{cccc}
0 & 0 & 0 & -i \\
0 & 0 & 0 & 0 \\
0 & 0 & 0 & 0 \\
i & 0 & 0 & 0
\end{array}\right) \text {, }
$$$$
\lambda_{11}=\left(\begin{array}{llll}
0 & 0 & 0 & 0 \\
0 & 0 & 0 & 1 \\
0 & 0 & 0 & 0 \\
0 & 1 & 0 & 0
\end{array}\right) \text {, }
$$$$
\lambda_{12}=\left(\begin{array}{cccc}
0 & 0 & 0 & 0 \\
0 & 0 & 0 & -i \\
0 & 0 & 0 & 0 \\
0 & i & 0 & 0
\end{array}\right) \text {, }
$$$$
\lambda_{13}=\left(\begin{array}{llll}
0 & 0 & 0 & 0 \\
0 & 0 & 0 & 0 \\
0 & 0 & 0 & 1 \\
0 & 0 & 1 & 0
\end{array}\right) \text {, }
$$$$
\lambda_{14}=\left(\begin{array}{cccc}
0 & 0 & 0 & 0 \\
0 & 0 & 0 & 0 \\
0 & 0 & 0 & -i \\
0 & 0 & i & 0
\end{array}\right) \text {, }
$$$$
\lambda_{15}=\frac{1}{\sqrt{6}}\left(\begin{array}{cccc}
1 & 0 & 0 & 0 \\
0 & 1 & 0 & 0 \\
0 & 0 & 1 & 0 \\
0 & 0 & 0 & -3
\end{array}\right) \text {. }
$$

The generators satisfy the following relation:

$$
T^{a} T^{b}=-\frac{1}{8} \delta^{a b} \mathbf{I}_{4}-\frac{1}{2} f^{a b c} T^{c}+\frac{i}{2} d^{a b c} T^{c}
$$

where the structure constants $f^{a b c}$ are completely antisymmetric while $d^{a b c}$ are symmetric with respect to all of their indices. Their values are shown up in the Tables 1 and 2. We have got these tables from [35]. 
TABLE 2: The nonvanishing structure constants $d^{a b c}$.

\begin{tabular}{|c|c|c|c|}
\hline$a$ & $b$ & $c$ & $d^{a b c}$ \\
\hline 1 & 1 & 8 & $1 / \sqrt{3}$ \\
\hline 1 & 1 & 15 & $1 / \sqrt{6}$ \\
\hline 1 & 4 & 6 & $1 / 2$ \\
\hline 1 & 5 & 7 & $1 / 2$ \\
\hline 1 & 9 & 11 & $1 / 2$ \\
\hline 1 & 10 & 12 & $1 / 2$ \\
\hline 2 & 2 & 8 & $1 / \sqrt{3}$ \\
\hline 2 & 2 & 15 & $1 / \sqrt{6}$ \\
\hline 2 & 4 & 7 & $-1 / 2$ \\
\hline 2 & 5 & 6 & $1 / 2$ \\
\hline 2 & 9 & 12 & $-1 / 2$ \\
\hline 2 & 10 & 11 & $1 / 2$ \\
\hline 3 & 3 & 8 & $1 / \sqrt{3}$ \\
\hline 3 & 3 & 15 & $1 / \sqrt{6}$ \\
\hline 3 & 4 & 4 & $1 / 2$ \\
\hline 3 & 5 & 5 & $1 / 2$ \\
\hline 3 & 6 & 6 & $-1 / 2$ \\
\hline 3 & 7 & 7 & $-1 / 2$ \\
\hline 3 & 9 & 9 & $1 / 2$ \\
\hline 3 & 10 & 10 & $1 / 2$ \\
\hline 3 & 11 & 11 & $-1 / 2$ \\
\hline 3 & 12 & 12 & $-1 / 2$ \\
\hline 4 & 4 & 8 & $-1 / 2 \sqrt{3}$ \\
\hline 4 & 4 & 15 & $1 / \sqrt{6}$ \\
\hline 4 & 9 & 13 & $1 / 2$ \\
\hline 4 & 10 & 14 & $1 / 2$ \\
\hline 5 & 5 & 8 & $-1 / 2 \sqrt{3}$ \\
\hline 5 & 5 & 15 & $1 / \sqrt{6}$ \\
\hline 5 & 9 & 14 & $-1 / 2$ \\
\hline 5 & 10 & 13 & $1 / 2$ \\
\hline 6 & 6 & 8 & $-1 / 2 \sqrt{3}$ \\
\hline 6 & 6 & 15 & $1 / \sqrt{6}$ \\
\hline 6 & 11 & 13 & $1 / 2$ \\
\hline 6 & 12 & 14 & $1 / 2$ \\
\hline 7 & 7 & 8 & $-1 / 2 \sqrt{3}$ \\
\hline 7 & 7 & 15 & $1 / \sqrt{6}$ \\
\hline 7 & 11 & 14 & $-1 / 2$ \\
\hline 7 & 12 & 13 & $1 / 2$ \\
\hline 8 & 8 & 8 & $-1 / \sqrt{3}$ \\
\hline 8 & 8 & 15 & $1 / \sqrt{6}$ \\
\hline 8 & 9 & 9 & $1 / 2 \sqrt{3}$ \\
\hline 8 & 10 & 10 & $1 / 2 \sqrt{3}$ \\
\hline 8 & 11 & 11 & $1 / 2 \sqrt{3}$ \\
\hline 8 & 12 & 12 & $1 / 2 \sqrt{3}$ \\
\hline 8 & 13 & 13 & $-1 / \sqrt{3}$ \\
\hline 8 & 14 & 14 & $-1 / \sqrt{3}$ \\
\hline 9 & 9 & 15 & $-1 / \sqrt{6}$ \\
\hline 10 & 10 & 15 & $-1 / \sqrt{6}$ \\
\hline 11 & 11 & 15 & $-1 / \sqrt{6}$ \\
\hline 12 & 12 & 15 & $-1 / \sqrt{6}$ \\
\hline 13 & 13 & 15 & $-1 / \sqrt{6}$ \\
\hline 14 & 14 & 15 & $-1 / \sqrt{6}$ \\
\hline 15 & 15 & 15 & $-\sqrt{2 / 3}$ \\
\hline
\end{tabular}

\section{B. Six-Dimensional 't Hooft Symbols}

The explicit representation of the six-dimensional 't Hooft symbol $\eta_{A B}^{a}=-\operatorname{Tr}\left(T^{a} J_{+}^{A B}\right)$ is given by

$$
\eta_{A B}^{1}=\frac{1}{2}\left(\begin{array}{cccccc}
0 & 0 & 0 & 1 & 0 & 0 \\
0 & 0 & 1 & 0 & 0 & 0 \\
0 & -1 & 0 & 0 & 0 & 0 \\
-1 & 0 & 0 & 0 & 0 & 0 \\
0 & 0 & 0 & 0 & 0 & 0 \\
0 & 0 & 0 & 0 & 0 & 0
\end{array}\right)=\frac{i}{2} \lambda_{2} \otimes \sigma^{1}
$$$$
\eta_{A B}^{2}=-\frac{1}{2}\left(\begin{array}{cccccc}
0 & 0 & 1 & 0 & 0 & 0 \\
0 & 0 & 0 & -1 & 0 & 0 \\
-1 & 0 & 0 & 0 & 0 & 0 \\
0 & 1 & 0 & 0 & 0 & 0 \\
0 & 0 & 0 & 0 & 0 & 0 \\
0 & 0 & 0 & 0 & 0 & 0
\end{array}\right)=-\frac{i}{2} \lambda_{2} \otimes \sigma^{3},
$$

$$
\begin{aligned}
\eta_{A B}^{3} & =\frac{1}{2}\left(\begin{array}{cccccc}
0 & 1 & 0 & 0 & 0 & 0 \\
-1 & 0 & 0 & 0 & 0 & 0 \\
0 & 0 & 0 & 1 & 0 & 0 \\
0 & 0 & -1 & 0 & 0 & 0 \\
0 & 0 & 0 & 0 & 0 & 0 \\
0 & 0 & 0 & 0 & 0 & 0
\end{array}\right) \\
& =\frac{i}{2}\left(\frac{2}{3} \mathbf{I}_{3}+\frac{1}{\sqrt{3}} \lambda_{8}\right) \otimes \sigma^{2},
\end{aligned}
$$$$
\eta_{A B}^{4}=\frac{1}{2}\left(\begin{array}{cccccc}
0 & 0 & 0 & 0 & 0 & 0 \\
0 & 0 & 0 & 0 & 0 & 0 \\
0 & 0 & 0 & 0 & 0 & 1 \\
0 & 0 & 0 & 0 & 1 & 0 \\
0 & 0 & 0 & -1 & 0 & 0 \\
0 & 0 & -1 & 0 & 0 & 0
\end{array}\right)=\frac{i}{2} \lambda_{7} \otimes \sigma^{1}
$$$$
\eta_{A B}^{5}=-\frac{1}{2}\left(\begin{array}{cccccc}
0 & 0 & 0 & 0 & 0 & 0 \\
0 & 0 & 0 & 0 & 0 & 0 \\
0 & 0 & 0 & 0 & 1 & 0 \\
0 & 0 & 0 & 0 & 0 & -1 \\
0 & 0 & -1 & 0 & 0 & 0 \\
0 & 0 & 0 & 1 & 0 & 0
\end{array}\right)=-\frac{i}{2} \lambda_{7} \otimes \sigma^{3},
$$$$
\eta_{A B}^{6}=\frac{1}{2}\left(\begin{array}{cccccc}
0 & 0 & 0 & 0 & 0 & 1 \\
0 & 0 & 0 & 0 & -1 & 0 \\
0 & 0 & 0 & 0 & 0 & 0 \\
0 & 0 & 0 & 0 & 0 & 0 \\
0 & 1 & 0 & 0 & 0 & 0 \\
-1 & 0 & 0 & 0 & 0 & 0
\end{array}\right)=\frac{i}{2} \lambda_{4} \otimes \sigma^{2},
$$ 


$$
\begin{aligned}
& \eta_{A B}^{7}=-\frac{1}{2}\left(\begin{array}{cccccc}
0 & 0 & 0 & 0 & 1 & 0 \\
0 & 0 & 0 & 0 & 0 & 1 \\
0 & 0 & 0 & 0 & 0 & 0 \\
0 & 0 & 0 & 0 & 0 & 0 \\
-1 & 0 & 0 & 0 & 0 & 0 \\
0 & -1 & 0 & 0 & 0 & 0
\end{array}\right)=-\frac{i}{2} \lambda_{5} \otimes \mathbf{I}_{2}, \quad \eta_{A B}^{13}=\frac{1}{2}\left(\begin{array}{cccccc}
0 & 0 & 0 & 1 & 0 & 0 \\
0 & 0 & -1 & 0 & 0 & 0 \\
0 & 1 & 0 & 0 & 0 & 0 \\
-1 & 0 & 0 & 0 & 0 & 0 \\
0 & 0 & 0 & 0 & 0 & 0 \\
0 & 0 & 0 & 0 & 0 & 0
\end{array}\right)=\frac{i}{2} \lambda_{1} \otimes \sigma^{2}, \\
& \eta_{A B}^{8}=-\frac{1}{2 \sqrt{3}}\left(\begin{array}{cccccc}
0 & 1 & 0 & 0 & 0 & 0 \\
-1 & 0 & 0 & 0 & 0 & 0 \\
0 & 0 & 0 & -1 & 0 & 0 \\
0 & 0 & 1 & 0 & 0 & 0 \\
0 & 0 & 0 & 0 & 0 & -2 \\
0 & 0 & 0 & 0 & 2 & 0
\end{array}\right) \quad \eta_{A B}^{14}=\frac{1}{2}\left(\begin{array}{cccccc}
0 & 0 & 1 & 0 & 0 & 0 \\
0 & 0 & 0 & 1 & 0 & 0 \\
-1 & 0 & 0 & 0 & 0 & 0 \\
0 & -1 & 0 & 0 & 0 & 0 \\
0 & 0 & 0 & 0 & 0 & 0 \\
0 & 0 & 0 & 0 & 0 & 0
\end{array}\right)=\frac{i}{2} \lambda_{2} \otimes \mathbf{I}_{2}, \\
& =-\frac{i}{2 \sqrt{3}}\left(-\frac{2}{3} \mathbf{I}_{3}+\lambda_{3}+\frac{2}{\sqrt{3}} \lambda_{8}\right) \otimes \sigma^{2}, \\
& \eta_{A B}^{9}=-\frac{1}{2}\left(\begin{array}{cccccc}
0 & 0 & 0 & 0 & 0 & 1 \\
0 & 0 & 0 & 0 & 1 & 0 \\
0 & 0 & 0 & 0 & 0 & 0 \\
0 & 0 & 0 & 0 & 0 & 0 \\
0 & -1 & 0 & 0 & 0 & 0 \\
-1 & 0 & 0 & 0 & 0 & 0
\end{array}\right)=-\frac{i}{2} \lambda_{5} \otimes \sigma^{1}, \\
& \begin{aligned}
\eta_{A B}^{15} & =\frac{1}{\sqrt{6}}\left(\begin{array}{cccccc}
0 & 1 & 0 & 0 & 0 & 0 \\
-1 & 0 & 0 & 0 & 0 & 0 \\
0 & 0 & 0 & -1 & 0 & 0 \\
0 & 0 & 1 & 0 & 0 & 0 \\
0 & 0 & 0 & 0 & 0 & 1 \\
0 & 0 & 0 & 0 & -1 & 0
\end{array}\right) \\
& =\frac{i}{\sqrt{6}}\left(\frac{1}{3} \mathbf{I}_{3}+\lambda_{3}-\frac{1}{\sqrt{3}} \lambda_{8}\right) \otimes \sigma^{2}
\end{aligned}
\end{aligned}
$$$$
\eta_{A B}^{10}=\frac{1}{2}\left(\begin{array}{cccccc}
0 & 0 & 0 & 0 & 1 & 0 \\
0 & 0 & 0 & 0 & 0 & -1 \\
0 & 0 & 0 & 0 & 0 & 0 \\
0 & 0 & 0 & 0 & 0 & 0 \\
-1 & 0 & 0 & 0 & 0 & 0 \\
0 & 1 & 0 & 0 & 0 & 0
\end{array}\right)=\frac{i}{2} \lambda_{5} \otimes \sigma^{3}
$$$$
\eta_{A B}^{11}=\frac{1}{2}\left(\begin{array}{cccccc}
0 & 0 & 0 & 0 & 0 & 0 \\
0 & 0 & 0 & 0 & 0 & 0 \\
0 & 0 & 0 & 0 & 0 & 1 \\
0 & 0 & 0 & 0 & -1 & 0 \\
0 & 0 & 0 & 1 & 0 & 0 \\
0 & 0 & -1 & 0 & 0 & 0
\end{array}\right)=\frac{i}{2} \lambda_{6} \otimes \sigma^{2}
$$$$
\eta_{A B}^{12}=-\frac{1}{2}\left(\begin{array}{cccccc}
0 & 0 & 0 & 0 & 0 & 0 \\
0 & 0 & 0 & 0 & 0 & 0 \\
0 & 0 & 0 & 0 & 1 & 0 \\
0 & 0 & 0 & 0 & 0 & 1 \\
0 & 0 & -1 & 0 & 0 & 0 \\
0 & 0 & 0 & -1 & 0 & 0
\end{array}\right)=-\frac{i}{2} \lambda_{7} \otimes \mathbf{I}_{2},
$$

where $\mathbf{I}_{n}$ is the $n$-dimensional identity matrix and $\left(\sigma^{1}, \sigma^{2}, \sigma^{3}\right)$ are the Pauli matrices and $\lambda_{\widehat{a}}(\widehat{a}=1, \ldots, 8)$ are the $S U(3)$ Gell-Mann matrices.

Another six-dimensional 't Hooft symbol $\bar{\eta}_{A B}^{a}=$ $-\operatorname{Tr}\left(\left(T^{a}\right)^{*} J_{-}^{A B}\right)$ can be obtained similarly:

$$
\begin{aligned}
& \bar{\eta}_{A B}^{1}=-\frac{i}{2} \lambda_{2} \otimes \sigma^{1}, \\
& \bar{\eta}_{A B}^{2}=-\frac{i}{2} \lambda_{2} \otimes \sigma^{3}, \\
& \bar{\eta}_{A B}^{3}=-\frac{i}{2}\left(\frac{2}{3} \mathbf{I}_{3}+\frac{1}{\sqrt{3}} \lambda_{8}\right) \otimes \sigma^{2}, \\
& \bar{\eta}_{A B}^{4}=\frac{i}{2} \lambda_{6} \otimes \sigma^{2}, \\
& \bar{\eta}_{A B}^{5}=-\frac{i}{2} \lambda_{7} \otimes \mathbf{I}_{2}, \\
& \bar{\eta}_{A B}^{6}=\frac{i}{2} \lambda_{5} \otimes \sigma^{1}, \\
& \bar{\eta}_{A B}^{7}=-\frac{i}{2} \lambda_{5} \otimes \sigma^{3}, \\
& \bar{\eta}_{A B}^{8}=\frac{i}{2 \sqrt{3}}\left(\frac{2}{3} \mathbf{I}_{3}+\lambda_{3}-\frac{2}{\sqrt{3}} \lambda_{8}\right) \otimes \sigma^{2}, \\
& \bar{\eta}_{A B}^{9}=-\frac{i}{2} \lambda_{4} \otimes \sigma^{2},
\end{aligned}
$$




$$
\begin{aligned}
& \bar{\eta}_{A B}^{10}=\frac{i}{2} \lambda_{5} \otimes \mathbf{I}_{2}, \\
& \bar{\eta}_{A B}^{11}=\frac{i}{2} \lambda_{7} \otimes \sigma^{1}, \\
& \bar{\eta}_{A B}^{12}=-\frac{i}{2} \lambda_{7} \otimes \sigma^{3}, \\
& \bar{\eta}_{A B}^{13}=-\frac{i}{2} \lambda_{1} \otimes \sigma^{2}, \\
& \bar{\eta}_{A B}^{14}=\frac{i}{2} \lambda_{2} \otimes \mathbf{I}_{2}, \\
& \bar{\eta}_{A B}^{15}=\frac{i}{\sqrt{6}}\left(\frac{1}{3} \mathbf{I}_{3}-\lambda_{3}-\frac{1}{\sqrt{3}} \lambda_{8}\right) \otimes \sigma^{2} .
\end{aligned}
$$

In order to derive the algebras obeyed by the 't Hooft symbols, first note that either Spin(6) generators $J_{+}^{A B}$ or $S U(4)$ generators $T_{+}^{a} \equiv T^{a}$ and $T_{-}^{a} \equiv\left(\mathrm{T}^{a}\right)^{*}$ can serve as a complete basis of any traceless, Hermitian $4 \times 4$ matrix $K$; that is,

$$
K=\sum_{a=1}^{15} k_{a}^{ \pm} T_{ \pm}^{a}=\frac{1}{2} \sum_{A, B=1}^{6} K_{A B}^{ \pm} J_{ \pm}^{A B} .
$$

Using definition (59), one can easily deduce that

$$
\begin{aligned}
T_{ \pm}^{a} & =\frac{1}{2} \eta_{A B}^{( \pm) a} J_{ \pm}^{A B}, \\
J_{ \pm}^{A B} & =2 \eta_{A B}^{( \pm) a} T_{ \pm}^{a},
\end{aligned}
$$

where $\eta_{A B}^{(+) a} \equiv \eta_{A B}^{a}$ and $\eta_{A B}^{(-) a} \equiv \bar{\eta}_{A B}^{a}$. Then one can consider the following matrix products:

$$
\begin{aligned}
\text { (I): } T_{ \pm}^{a} T_{ \pm}^{b} & =\frac{1}{4} \eta_{A B}^{( \pm) a} \eta_{C D}^{( \pm) b} J_{ \pm}^{A B} J_{ \pm}^{C D}, \\
\text { (II): } J_{ \pm}^{A B} J_{ \pm}^{C D} & =4 \eta_{A B}^{( \pm) a} \eta_{C D}^{( \pm) b} T_{ \pm}^{a} T_{ \pm}^{b} .
\end{aligned}
$$

By applying (11), (A.5), and (A.9) to the above matrix products, one can easily get the algebras obeyed by the sixdimensional 't Hooft symbols:

$$
\begin{aligned}
& \eta_{A B}^{a} \eta_{A B}^{b}=\delta^{a b}=\bar{\eta}_{A B}^{a} \bar{\eta}_{A B}^{b}, \\
& \eta_{A B}^{a} \eta_{C D}^{a}=\frac{1}{2}\left(\delta_{A C} \delta_{B D}-\delta_{A D} \delta_{B C}\right)=\bar{\eta}_{A B}^{a} \bar{\eta}_{C D}^{a}, \\
& \frac{1}{4} \varepsilon^{A B C D E F} \eta_{C D}^{a} \eta_{E F}^{b}=d^{a b c} \eta_{A B}^{c}, \\
& \frac{1}{4} \varepsilon^{A B C D E F} \bar{\eta}_{C D}^{a} \bar{\eta}_{E F}^{b}=d^{a b c} \bar{\eta}_{A B}^{c}, \\
& \eta_{A C}^{a} \eta_{B C}^{b}-\eta_{B C}^{a} \eta_{A C}^{b}=f^{a b c} \eta_{A B}^{c}, \\
& \bar{\eta}_{A C}^{a} \bar{\eta}_{B C}^{b}-\bar{\eta}_{B C}^{a} \bar{\eta}_{A C}^{b}=f^{a b c} \bar{\eta}_{A B}^{c}, \\
& f^{a b c} \eta_{A B}^{a} \eta_{C D}^{b} \\
& \quad=\frac{1}{2}\left(\delta_{A C} \eta_{B D}^{c}-\delta_{A D} \eta_{B C}^{c}-\delta_{B C} \eta_{A D}^{c}+\delta_{B D} \eta_{A C}^{c}\right),
\end{aligned}
$$

$$
\begin{aligned}
& f^{a b c} \bar{\eta}_{A B}^{a} \bar{\eta}_{C D}^{b} \\
& \quad=\frac{1}{2}\left(\delta_{A C} \bar{\eta}_{B D}^{c}-\delta_{A D} \bar{\eta}_{B C}^{c}-\delta_{B C} \bar{\eta}_{A D}^{c}+\delta_{B D} \bar{\eta}_{A C}^{c}\right), \\
& d^{a b c} \eta_{A B}^{a} \eta_{C D}^{b}=\frac{1}{4} \varepsilon^{A B C D E F} \eta_{E F}^{c}, \\
& d^{a b c} \bar{\eta}_{A B}^{a} \bar{\eta}_{C D}^{b}=\frac{1}{4} \varepsilon^{A B C D E F} \bar{\eta}_{E F}^{c} .
\end{aligned}
$$

Finally we list the nonzero components of the 't Hooft symbols in the basis of complex coordinates $z^{\alpha}=\left\{z^{1}=\right.$ $\left.x^{1}+i x^{2}, z^{2}=x^{3}+i x^{4}, z^{3}=x^{5}+i x^{6}\right\}$ and their complex conjugates $\bar{z}^{\bar{\alpha}}$ where $\alpha, \bar{\alpha}=1,2,3$. We will denote $\eta_{\alpha \beta}^{a}=\eta_{z^{\alpha} z^{\beta}}^{a}$, $\eta_{\alpha \bar{\beta}}^{a}=\eta_{z^{\alpha} \bar{z}_{\bar{\beta}}}^{a}$, and so forth in the hope of no confusion with the real basis:

$$
\begin{aligned}
& \eta_{12}^{1}=-\frac{i}{4} \\
& \eta_{12}^{2}=-\frac{1}{4} \\
& \eta_{23}^{4}=-\frac{i}{4} \\
& \eta_{23}^{5}=-\frac{1}{4} \\
& \eta_{13}^{9}=\frac{i}{4} \\
& \eta_{13}^{10}=\frac{1}{4} \\
& \eta_{1 \overline{2}}^{14}=\frac{1}{4} \\
& \eta_{1 \overline{1}}^{3}=\frac{i}{4} \\
& \eta_{2 \overline{3}}^{12}=-\frac{1}{4} \\
& \eta_{2 \overline{2}}^{3}=\frac{i}{4} \\
& \eta_{2 \overline{3}}^{11}=\frac{i}{4} \\
& \eta_{1 \overline{3}}^{6}=\frac{i}{4} \\
& \eta_{1 \overline{3}}^{7}=-\frac{1}{4} \\
& \frac{i}{4}
\end{aligned}
$$




$$
\begin{aligned}
& \eta_{1 \overline{1}}^{15}=\frac{i}{2 \sqrt{6}}, \\
& \eta_{2 \overline{2}}^{15}=-\frac{i}{2 \sqrt{6}}, \\
& \eta_{3 \overline{3}}^{15}=\frac{i}{2 \sqrt{6}} .
\end{aligned}
$$

Here the complex conjugates are not shown up since they can easily be implemented. The corresponding values of $\bar{\eta}_{A B}^{a}$ can be obtained from those of $\eta_{A B}^{a}$ by flipping the sign for the entries $a=1,3,4,6,8,9,11,13,15$ as well as interchanging $z^{3} \leftrightarrow \bar{z}^{3}$ for all entries. Note that the first line in (B.17) belongs to $m_{A B}^{(+) \dot{a}}$ in (77) with purely holomorphic or antiholomorphic indices. This result implies that the space of complex structure deformations can be identified with the coset space $S U(4) / U(3)=\mathbb{C} P^{3}[9]$.

\section{Conflicts of Interest}

The authors declare that they have no conflicts of interest.

\section{Acknowledgments}

The authors are grateful to Chanju Kim for collaboration at an early stage and very helpful discussions. Hyun Seok Yang thanks Richard Szabo for his warm hospitality and valuable discussions during his visit to Heriot-Watt University, Edinburgh. He also thanks Jungjai Lee and John J. Oh for constructive discussions and collaborations with a related subject. The work of $\mathrm{H}$. S. Yang was supported by the National Research Foundation of Korea (NRF) grant funded by the Korean government (MOE) (no. NRF2015R1D1A1A01059710). The work of S. Yun was supported by the World Class University Grant no. R32-10130.

\section{References}

[1] M. B. Green, J. H. Schwarz, and E. Witten, Superstring Theory, vol. 1, Cambridge University Press, Cambridge, Uk, 1987.

[2] P. Candelas, G. T. Horowitz, A. Strominger, and E. Witten, "Vacuum configurations for superstrings," Nuclear Physics B, vol. 258, no. 1, pp. 46-74, 1985.

[3] K. Hori, S. Katz, A. Klemm et al., Mirror Symmetry, American Mathematical Society and the Clay Mathematics Institute, Cambridge, Mass, USA, 2003.

[4] E. Witten, "Mirror manifolds and topological field theory," in Essays on Mirror Manifolds, S.-T. Yau, Ed., pp. 120-158, International Press, Somerville, Mass, USA, 1992.

[5] M. Kontsevich, Homological algebra of mirror symmetry, [ alggeom/9411018],

[6] "Symplectic geometry and mirror symmetry," in Proceedings of the 4th KIAS Annual International Conference, K. Fukaya, Y.-G. Oh, K. Ono, and G. Tian, Eds., World Scientific, Seoul, South Korea, August 2000.

[7] A. Strominger, S.-T. Yau, and E. Zaslow, "Mirror symmetry is Tduality," Nuclear Physics B, vol. 479, no. 1-2, pp. 243-259, 1996.
[8] W. K. Misner, S. Thorne, and J. A . Wheeler, Gravitation, W. H. Freeman and Company, New York, NY, USA, 1973.

[9] H. B. Lawson and M.-L. Michelsohn, Spin Geometry, Princeton University Press, Princeton, NJ, USA, 1989.

[10] E. Meinrenken, Clifford Algebras and Lie Theory, vol. 58, Springer, Berlin, Germany, 2013.

[11] J. J. Oh, C. Park, and H. S. Yang, "Yang-Mills instantons from gravitational instantons," Journal of High Energy Physics, vol. 4, article 87, 2011.

[12] J. J. Oh and H. S. Yang, "Einstein manifolds as Yang-Mills instantons," Modern Physics Letters A, vol. 28, no. 21, 2013.

[13] J. Lee, J. J. Oh, and H. S. Yang, "An efficient representation of Euclidean gravity I," Journal of High Energy Physics, vol. 12, article 25, 2011.

[14] H. S. Yang, "Riemannian manifolds and gauge theory," in Proceedings of the Corfu Summer Institute 2011 PoS (CORFU '11), Corfu, Greece, September 2011.

[15] M. F. Atiyah, N. J. Hitchin, and I. M. Singer, "Self-duality in fourdimensional Riemannian geometry," Proceedings of the Royal Society A Mathematical, Physical and Engineering Sciences, vol. 362, no. 1711, pp. 425-461, 1978.

[16] A. L. Besse, Einstein Manifolds, Springer, Berlin, Germany, 1987.

[17] S. K. Donaldson, "Anti self-dual Yang-Mills connections over complex algebraic surfaces and stable vector bundles," Proceedings of the London Mathematical Society, vol. 50, no. 1, pp. 1-26, 1985.

[18] K. Uhlenbeck and S.-T. Yau, "On the existence of HermitianYang-Mills connections in stable vector bundles," Communications on Pure and Applied Mathematics, vol. 39, pp. S257-S293, 1986.

[19] S.-T. Yau and S. Nadis, The Shape of Inner Space, Basic Books, New York, NY, USA, 2010.

[20] R. Slansky, "Group theory for unified model building," Physics Reports, vol. 79, no. 1, pp. 1-128, 1981.

[21] R. Bott and L. W. Tu, Differential Forms in Algebraic Topology, vol. 82 of Graduate Texts in Mathematics, Springer, New York, NY, USA, 1982.

[22] G. Tian, "Gauge theory and calibrated geometry, I," Annals of Mathematics, vol. 151, no. 1, pp. 193-268, 2000.

[23] M. R. Douglas, R. Reinbacher, and S.-T. Yau, "Branes, bundles and attractors: bogomolov and beyond," Algebraic Geometry, vol. 4, 2006.

[24] A. Strominger, "Superstrings with torsion," Nuclear Physics B, vol. 274, no. 2, pp. 253-284, 1986.

[25] J. Li and S.-T. Yau, "The existence of supersymmetric string theory with torsion," Journal of Differential Geometry, vol. 70, no. 1, pp. 143-181, 2005.

[26] L. B. Anderson, J. Gray, A. Lukas, and B. Ovrut, "The edge of supersymmetry: stability walls in heterotic theory," Physics Letters B, vol. 677, no. 3-4, pp. 190-194, 2009.

[27] I. Smith, R. P. Thomas, and S.-T. Yau, "Symplectic conifold transitions," Journal of Differential Geometry, vol. 62, no. 2, pp. 209-242, 2002.

[28] H. S. Yang, "Towards a background independent quantum gravity," Journal of Physics: Conference Series, vol. 343, no. 1, 2012.

[29] H. S. Yang, "Quantization of emergent gravity," International Journal of Modern Physics A, vol. 30, no. 4-5, 115 pages, 2015.

[30] H. S. Yang, "Highly effective action from large $N$ gauge fields," Physical Review D, vol. 90, Article ID 086006, 2014. 
[31] H. S. Yang, "Calabi-Yau manifolds from noncommutative Hermitian U(1) instantons," Physical Review D, vol. 91, no. 10, Article ID 104002, 104002 pages, 2015.

[32] M. Mariño, R. Minasian, G. Moore, and A. Strominger, "Nonlinear instantons from supersymmetric $p$-branes," Journal of High Energy Physics, vol. 1, article 5, 2000.

[33] H. S. Yang, "Mirror symmetry in emergent gravity," Nuclear Physics B, vol. 922, pp. 264-279, 2017.

[34] G. Arutyunov and S. Frolov, "Foundations of the $\mathrm{AdS}_{5} \times S^{5}$ superstring: I," Journal of Physics A: Mathematical and General, vol. 42, no. 25, 2009.

[35] W. Greiner and B. Müller, Quantum Mechanics: Symmetries, Springer-Verlag, Berlin, Germany, 1993. 

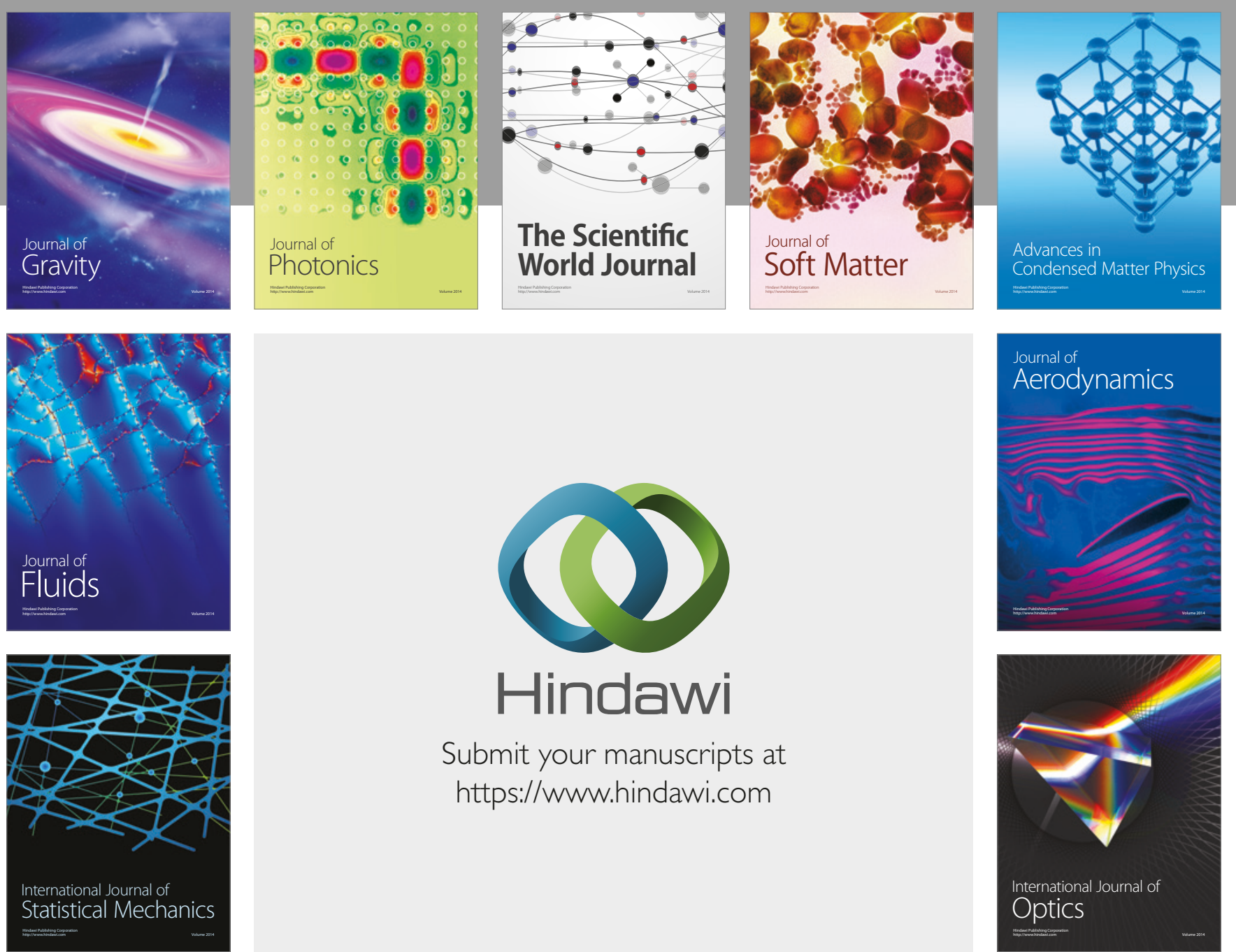

Submit your manuscripts at

https://www.hindawi.com
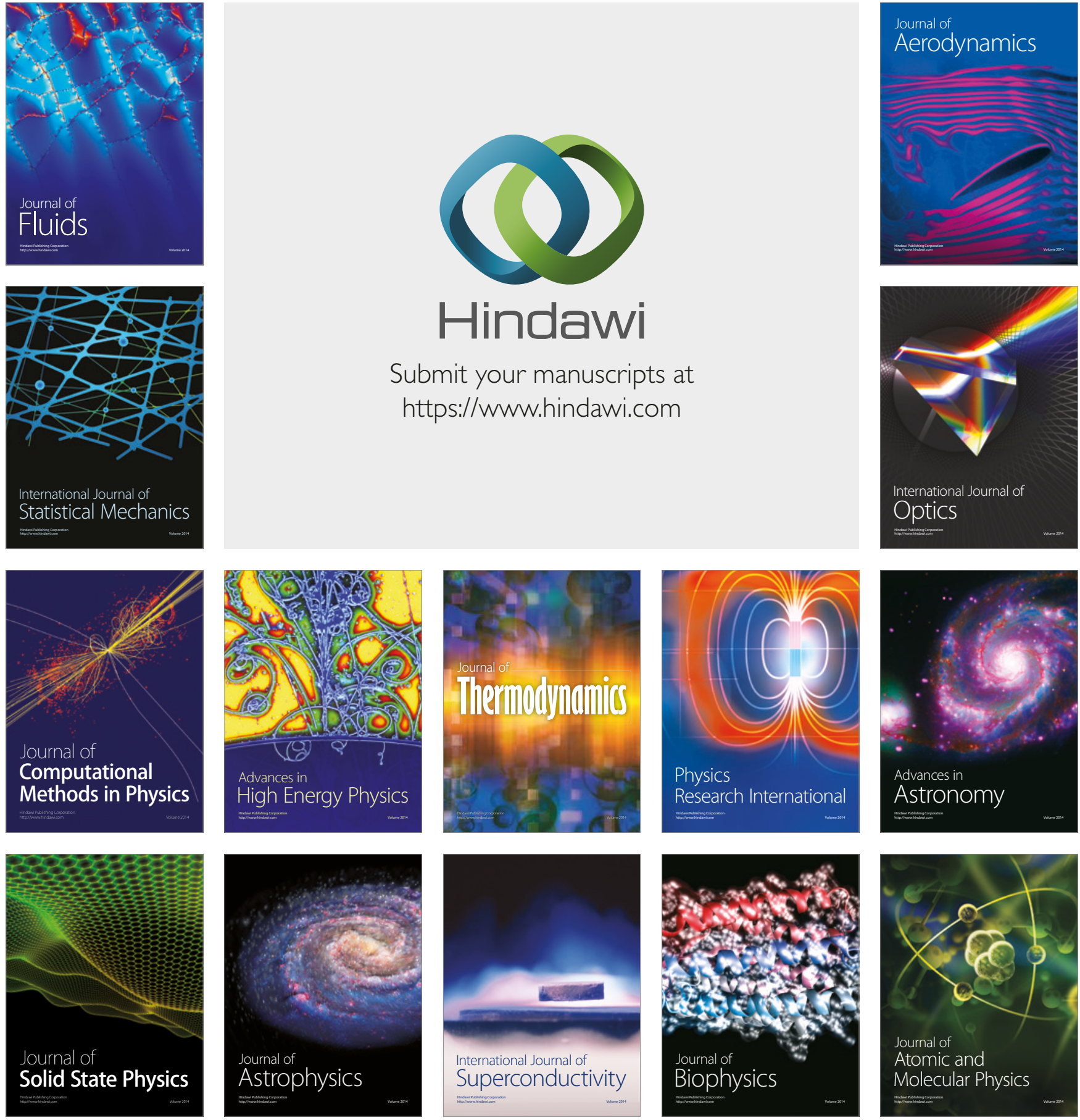